\title{
RADIATIVE AND AUGER DECAY DATA FOR MODELING NICKEL K LINES
}

\author{
P. PALMERI \\ Astrophysique et Spectroscopie, Université de Mons-Hainaut, B-7000 Mons, Belgium; palmeri@umh.ac.be \\ P. QuineT \\ Astrophysique et Spectroscopie, Université de Mons-Hainaut, B-7000 Mons, Belgium; and IPNAS, Sart Tilman B15, \\ Université de Liège, B-4000 Liège, Belgium; quinet@umh.ac.be \\ C. Mendoza \\ Centro de Física, IVIC, Caracas 1020A, Venezuela; claudio@ivic.ve \\ M. A. Bautista \\ Department of Physics, Virginia Polytechnic and State University, Blacksburg, VA 24061; bautista@vt.edu \\ J. GARCía \\ IACS-Department of Physics, The Catholic University of America, Washington, DC 20064; javier@milkyway.gsfc.nasa.gov \\ AND \\ M. C. Witthoeft and T. R. Kallman \\ NASA Goddard Space Flight Center, Greenbelt, MD 20771; michael.c.witthoeft@nasa.gov; timothy.r.kallman@nasa.gov \\ Received 2008 June 6; accepted 2008 July 16
}

\begin{abstract}
Radiative and Auger decay data have been calculated for modeling the $\mathrm{K}$ lines in ions of the nickel isonuclear sequence, from $\mathrm{Ni}^{+}$up to $\mathrm{Ni}^{27+}$. Level energies, transition wavelengths, radiative transition probabilities, and radiative and Auger widths have been determined using Cowan's Hartree-Fock with relativistic corrections (HFR) method. Auger widths for the third-row ions $\left(\mathrm{Ni}^{+}-\mathrm{Ni}^{10+}\right)$ have been computed using single-configuration average (SCA) compact formulae. Results are compared with data sets computed with the AUTOSTRUCTURE and MCDF atomic structure codes and with available experimental and theoretical values, mainly in highly ionized ions and in the solid state.
\end{abstract}

Subject headings: atomic data - atomic processes — line: formation - X-rays: general

Online material: machine-readable tables

\section{INTRODUCTION}

The nickel $\mathrm{K}$ lines have arguable diagnostic potential in X-ray astronomy. They reside in a relatively unconfused part of the X-ray spectrum where they can be used to estimate quantities of interest such as the redshift, temperature, abundance, and the velocity of the emitting gas. Sensitive observations from the currently active $S u z a k u$ satellite, and those to be expected from future space missions, e.g., the Constellation- $X$ observatory, are bringing about further spectroscopic attention to the Ni K features.

On the other hand, clear detections of nickel have been obtained only in a few astrophysical X-ray spectra. This is undoubtedly due to a combination of limited counting statistics at energies above $7 \mathrm{keV}$, where the nickel lines occur, and to poor spectral resolution in this band, which makes nickel $\mathrm{K} \alpha$ and iron $\mathrm{K} \beta$ lines indistinguishable. The first detection of $\mathrm{Ni} \mathrm{K} \alpha$ in an X-ray binary was reported by Sako et al. (1999) in the Advanced Satellite for Cosmology and Astrophysics (ASCA) spectrum of Vela X-1. Fluorescence line energies and intensities were measured mostly for neutral elements, including nickel in eight ionization stages in spite of the uncertainties in the line energy $\left(7.52_{-0.05}^{+0.06} \mathrm{keV}\right)$. This detection was later confirmed by Goldstein et al. (2004) using three data sets obtained with the Chandra High Energy Transmission Grating Spectrometer (HETGS) during a binary orbit, representing the orbital eclipse phases of $\phi=0,0.25$, and 0.5 . They found relative strong $\mathrm{Ni} \alpha \alpha$ emission with an increase in the line flux of a factor of 15 between the $\phi=0$ and 0.5 phases.
X-ray absorption features from Ni have been found in X-ray binary systems. Boirin et al. (2004) presented evidence of $\mathrm{K} \alpha$ absorption in Ni XXVII at $7.82 \mathrm{keV}$ in the XMM-Newton spectrum of the low-mass X-ray binary (LMXB) XB 1916-053. In the case of the LMXB GX 13+1, Sidoli et al. (2002) came across two absorption features at 7.85 and $8.26 \mathrm{keV}$ which they respectively identified as either $\mathrm{K} \beta$ lines from $\mathrm{Fe} \mathrm{xxV}$ and $\mathrm{Fe}$ XXVI or $\mathrm{K} \alpha$ lines from Ni xxvII and Ni xxviII.

$\mathrm{Ni} \mathrm{K} \alpha$ emission in an active galactic nucleus (AGN) was first detected in the XMM-Newton X-ray spectra of the Circinus galaxy (Molendi et al. 2003). $\mathrm{Fe} \mathrm{K} \alpha$, $\mathrm{Fe} \mathrm{K} \beta$, and $\mathrm{Ni} \mathrm{K} \alpha$ were identified at 6.4, 7.058, and $7.472 \mathrm{keV}$, respectively. From the $\mathrm{Ni}$ and $\mathrm{Fe}$ $\mathrm{K} \alpha$ line fluxes, a nickel-to-iron abundance ratio was estimated at $0.055-0.075$, a factor of 1.5-2 larger than the cosmic values (Anders \& Grevesse 1989). Similar studies of the Seyfert 2 galaxy NGC 1068 were reported by Matt et al. (2004) using Chandra and $X M M$-Newton observations. $\mathrm{Fe}$ and Ni fluorescence emission in both neutral and highly ionized material was identified, and from the nickel-to-iron flux ratio, $\mathrm{Fe}$ was found to be overabundant by a factor of 2 with respect to solar and $\mathrm{Ni}$ by the same factor with respect to iron. Pounds \& Vaughan (2006) confirmed the nickel emission in NGC 1068 as well as in Mrk 3, a Seyfert 2 galaxy previously studied by Pounds \& Page (2005). Pounds \& Vaughan (2006) also showed evidence of high-velocity shifts in the lines coming from ionized nickel. Markowitz et al. (2007) detected with Suzaku narrow $\mathrm{K} \alpha$ fluorescence emission lines from $\mathrm{Fe}, \mathrm{Si}, \mathrm{S}, \mathrm{Ar}$, $\mathrm{Ca}$, and $\mathrm{Ni}$ in the radio-loud AGN Centaurus A (NGC 5128). 
Molendi et al. (1998) analyzed data collected with BeppoSAX from the Perseus cluster of galaxies, to find that the ratio of the flux of the 7-8 keV line complex to the $6.7 \mathrm{keV}$ line was significantly larger than that predicted by optically thin plasma codes and that it diminishes with increasing cluster radius. It is argued that this effect is due to resonance scattering in an optically thick plasma at the energies of the $\mathrm{Fe} \mathrm{K} \alpha$ line. However, Gastaldello $\&$ Molendi (2004) used XMM-Newton observations of the same cluster to measure an overabundance for nickel. These authors claimed that the excess in the flux of the 7-8 keV line complex was due to $\mathrm{Ni} \mathrm{K} \alpha$ emission rather than resonance scattering. De Plaa et al. (2004) also detected an overabundant nickel in the XMM-Newton spectrum of the cluster Abel 478, but they noticed that this could be overestimated due to errors in the nickel line energies. The determination of the Ni abundance profile in the intercluster medium is also important, because $\mathrm{Ni}$ is almost exclusively produced in Type Ia supernovae; therefore, highresolution spectral analysis, together with accurate atomic data, are needed to understand these observations.

The first object detected by the International Gamma-Ray Astrophysics Laboratory (INTEGRAL) in the Galactic plane, IGR J16318-4848, is a possible X-ray binary where the obscuring matter has a column density as large as the inverse Thompson cross section. This source also shows strong $\mathrm{Fe} \mathrm{K} \alpha$ and $\mathrm{Fe} \mathrm{K} \beta$ emission accompanied by a weaker, but nevertheless distinct, $\mathrm{Ni} \mathrm{K} \alpha$ feature at $7.5 \mathrm{keV}$ (Walter et al. 2003). Observations of the same source with XMM-Newton reveal similar properties (Matt \& Guainazzi 2003), and a subsequent monitoring campaign with both INTEGRAL and XMM-Newton confirmed column densities of $1.2 \times 10^{24} \mathrm{~cm}^{-2}$ and large equivalent widths for the K $\alpha$ emission lines of $\mathrm{Fe}$ and $\mathrm{Ni}$ (Ibarra et al. 2007).

Perhaps the first unambiguous detection of $\mathrm{Ni} \mathrm{K}$ lines in an astrophysical spectrum is due to Koyama et al. (2007). They have observed the diffuse X-ray emission from the Galactic center using the X-ray Imaging Spectrometer (XIS) on Suzaku. They have detected, for the first time, lowly ionized nickel and He-like nickel $\mathrm{K}$ lines and have measured the $\mathrm{K} \alpha$ line flux ratio of Ni xxvII to that of $\mathrm{Fe} x \mathrm{xv}$ to determine a plasma temperature of $\sim 5.4 \mathrm{keV}$, assuming solar relative $\mathrm{Ni}$ and $\mathrm{Fe}$ abundances.

Following work by Palmeri et al. (2002, 2003a, 2003b), Bautista et al. (2003, 2004), Mendoza et al. (2004), and Kallman et al. (2004) on the Fe K lines, by García et al. (2005) on the K-shell photoabsorption of $\mathrm{O}$ ions, and the recent study of the $\mathrm{K}$ lines in the $\mathrm{Ne}, \mathrm{Mg}, \mathrm{Si}, \mathrm{Ar}$, and $\mathrm{Ca}$ isonuclear sequences (Palmeri et al. 2008), we report new atomic data for K-vacancy levels in the nickel isonuclear sequence. Prime objectives are to improve the atomic database of the XSTAR modeling code (Bautista $\&$ Kallman 2001) and to prepare ionic targets (configuration expansions and orbitals) for the lengthy computations of the K-shell photoabsorption and photoionization cross sections, where both radiative and Auger dampings are key effects. With respect to $\mathrm{Ni}$, available atomic structure data sets, namely K-vacancy level energies, wavelengths, $A$-values, and radiative and Auger widths, for first-row ions with electron number $2 \leq N \leq 9$ are far from complete; while for the second and third-row ions $(10 \leq N \leq$ 27), they are certainly lacking. The only exception is $\mathrm{Ni}^{+}$, where measurements in the solid state have been reported (Salem \& Wimmer 1970; Salem et al. 1972; Slivinsky \& Ebert 1972; Berenyi et al. 1978; Rao et al. 1986; Perujo et al. 1987; Hölzer et al. 1997; Raj et al. 2001; Egri et al. 2008) and the K $\alpha$ unresolved transition array (UTA) centroid wavelength have been calculated by House (1969).

Previous study on the K-shell structure of nickel includes that by Hsuan et al. (1987) on the satellite spectra of He-like nickel.
They have recorded spectra emitted from the plasma of the Tokamak Fusion Test Reactor (TFTR) with a high-resolution crystal spectrometer, providing a K-line list for Ni XXVII $(N=2)$ and $\mathrm{Ni} \operatorname{xxvI}(N=3)$ and interpreting the observed spectra with the aid of a Hartree-Fock-Slater (HFS) calculation. Bombarda et al. (1988) have measured the wavelengths of the $\mathrm{K}$ lines in Ni xxIV-Ni xxviI $(N=2-5)$ emitted by a hot plasma at the Joint European Torus (JET). In order to analyze these observations, they have computed wavelengths, $A$-values, and radiative widths with the SUPERSTRUCTURE atomic structure code (Eissner et al. 1974) and Auger rates with AUTOLSJ (TFR Group et al. 1982). Vainshtein \& Safronova (1978) have calculated wavelengths, radiative transition probabilities, and autoionization (Auger) rates for ions with atomic numbers $Z=4-34$ using the $1 / Z$ expansion technique. They have considered the $1 s-2 p$ transitions in the H-like sequence, $1 s 2 l-2 p 2 l$ and $1 s^{2}-1 s 2 l$ in the He-like sequence, and $1 s^{2} 2 l-1 s 2 p 2 l$ in the Li-like sequence. Energies for K-vacancy levels of the type $1 \mathrm{snl}$ in the He-like isoelectronic sequence have been calculated by Vainshtein \& Safronova (1985) using the same technique. Gorczyca et al. (2003) have audited the fluorescence database by Kaastra \& Mewe (1993), which is widely used in modeling codes, in particular their scaling procedures along isoelectronic sequences. They have found serious flaws which appear to compromise the application of this database in plasma modeling.

The outline of the present report is as follows. The numerical methods are briefly described in $\S 2$, while an analysis of the results based on comparisons with previous experimental and theoretical values is carried out in $\S 3$. The two supplementary electronic tables are explained in $\S 4$, while some conclusions are finally discussed in $\S 5$.

\section{NUMERICAL METHODS}

Three independent atomic structure packages have been used. The main body of atomic data is computed with the Hartree-Fock with relativistic corrections (HFR) method of Cowan (1981). Data accuracy is assessed by means of two other approaches: the multiconfiguration Breit-Pauli method, which incorporates a scaled Thomas-Fermi-Dirac statistical potential as implemented in AUTOSTRUCTURE (Eissner et al. 1974; Badnell 1986, 1997), and the GRASP code (Grant et al. 1980; Grant \& McKenzie 1980; McKenzie et al. 1980), based on the multiconfiguration Dirac-Fock method.

In HFR and AUTOSTRUCTURE, wave functions are calculated with the Breit-Pauli relativistic corrections

$$
H_{\mathrm{BP}}=H_{\mathrm{NR}}+H_{1 \mathrm{~B}}+H_{2 \mathrm{~B}},
$$

where $H_{\mathrm{NR}}$ is the usual nonrelativistic Hamiltonian. The onebody relativistic operators

$$
H_{1 \mathrm{~B}}=\sum_{n=1}^{N} f_{n}(\operatorname{mass})+f_{n}(D)+f_{n}(\mathrm{so})
$$

represent the spin-orbit interaction, $f_{n}$ (so), the non-fine-structure mass variation, $f_{n}$ (mass), and one-body Darwin correction, $f_{n}(D)$. The two-body Breit operators are given by

$$
\begin{gathered}
H_{2 \mathrm{~B}}=\sum_{n<m} g_{n m}(\mathrm{so})+g_{n m}(\mathrm{ss})+g_{n m}(\mathrm{css}) \\
+g_{n m}(\mathrm{D})+g_{n m}(\mathrm{oo}),
\end{gathered}
$$


where the fine-structure terms are $g_{n m}$ (so) (spin-other-orbit and mutual spin-orbit) and $g_{n m}$ (ss) (spin-spin), and the non-finestructure counterparts are $g_{n m}(\mathrm{css})$ (spin-spin contact), $g_{n m}(\mathrm{D})$ (two-body Darwin), and $g_{n m}$ (oo) (orbit-orbit). HFR computes energies, $A$-values, and Auger rates with nonorthogonal orbital bases, which are generated by optimizing the average energy of each configuration. It also neglects the part of the Breit interaction (eq. [3]) that cannot be reduced to a one-body operator. AUTOSTRUCTURE can use both orthogonal and nonorthogonal orbital bases for all the electronic configurations considered, which enables estimates of relaxation effects. We have used the same configuration-interaction (CI) expansions as in our previous papers on the Fe isonuclear sequence (Bautista et al. 2003; Palmeri et al. 2003a, 2003b; Mendoza et al. 2004).

Auger rates are computed in both HFR and AUTOSTRUCTURE in a distorted wave approach. However, the level-to-level computation of Auger rates in ions with open $3 d$ shell $(N>17)$ proved to be very lengthy. Moreover, certain important Auger decay channels, such as $[1 s] 3 d^{5} \rightarrow[2 p 3 p] 3 d^{5} \varepsilon d$ in $\mathrm{Ni}^{6+}(N=22)$, did not get through either an HFR or an AUTOSTRUCTURE run. Therefore, we have employed the formula given in Palmeri et al. (2001) for the single-configuration average (SCA) Auger decay rate in order to compute the rates for these ions as we did in the $\mathrm{Fe}$ isonuclear sequence (Palmeri et al. 2003b). It was also verified in $\mathrm{Ni}^{9+}(N=19)$ and in $\mathrm{Ni}^{8+}(N=20)$ that the Auger widths (the total, KLL, KLM and KMM channels) change by less than a percent from K-vacancy fine-structure level to another and that single-configuration AUTOSTRUCTURE fine-structure calculations agree within $\sim 15 \%$ with HFR SCA calculations.

Our third package is GRASP where the atomic state function (ASF) is represented as a superposition of configuration state functions (CSF) of the type

$$
\Psi(\alpha \Pi J M)=\sum_{i} c_{i}(\alpha) \Phi\left(\beta_{i} \Pi J M\right)
$$

where $\Psi$ and $\Phi$ are, respectively, the ASF and CSF; $\Pi, J$, and $M$ are the relevant quantum numbers: parity, total angular momentum, and its associated total magnetic number, respectively; $\alpha$ and $\beta_{i}$ stand for all the other quantum numbers that are necessary to describe unambiguously the ASFs and CSFs. The summation in equation (4) is up to $n_{c}$, the number of CSFs in the expansion, and each CSF is built from antisymmetrized products of relativistic spin orbitals. The $c_{i}$ coefficients, together with the orbitals, are optimized by minimizing an energy functional. The latter is built from one or more eigenvalues of the Dirac-Coulomb Hamiltonian depending on the optimization option adopted. Here, we have used the extended average level (EAL) option in which the $(2 J+1)$ weighted trace of the Hamiltonian is minimized. Transverse Breit interaction as well as other QED interactions, e.g., the vacuum polarization and self-energy, have been included in the Hamiltonian matrix as perturbations. This code does not treat the continuum and has thus been exclusively employed in comparisons of radiative data for bound-bound transitions.

\section{RESULTS}

Detailed comparisons with previous data have been carried out in order to obtain accuracy estimates and detect weak points. In the following sections, we give a concise account of our HFR computations of level energies, radiative and Auger widths of $\mathrm{K}$-vacancy states, and wavelengths and radiative transition probabilities for $\mathrm{K}$ lines. With respect to the $\mathrm{K} \alpha_{1,2}$ and $\mathrm{K} \beta$ unresolved transition arrays (UTAs) characteristic in second- and third-row ions $(12 \leq N \leq 27)$, we have determined centroid wavelengths
TABLE 1

$K$-Vacancy Level Energies for Ni Ions with $2 \leq N \leq 3$

\begin{tabular}{|c|c|c|c|c|}
\hline$N$ & Level $^{\mathrm{a}}$ & $\begin{array}{l}\mathrm{NIST}^{\mathrm{a}} \\
(\mathrm{keV})\end{array}$ & $\begin{array}{l}\mathrm{HFR}^{\mathrm{b}} \\
(\mathrm{keV})\end{array}$ & $\begin{array}{c}\mathrm{VS}^{\mathrm{c}} \\
(\mathrm{keV})\end{array}$ \\
\hline 2. & $1 s 2 s^{3} S_{1}$ & 7.7316132 & 7.7331 & 7.7314 \\
\hline 2. & $1 s 2 p^{3} P_{0}^{o}$ & 7.7633680 & 7.7623 & 7.7631 \\
\hline $2 .$. & $1 s 2 p^{3} P_{1}^{o}$ & 7.7656952 & 7.7668 & 7.7657 \\
\hline 2. & $1 s 2 p^{3} P_{2}^{o}$ & 7.7864167 & 7.7876 & 7.7862 \\
\hline 2. & $1 s 2 s^{1} S_{0}$ & 7.7660015 & 7.7659 & 7.7658 \\
\hline 2 . & $1 s 2 p{ }^{1} P_{1}^{o}$ & 7.8055847 & 7.8073 & 7.8052 \\
\hline 3. & $1 s 2 s^{2}{ }^{2} S_{1 / 2}$ & 7.6933430 & 7.6944 & \\
\hline $3 \ldots$ & $1 s\left({ }^{2} S\right) 2 s 2 p\left({ }^{3} P^{o}\right){ }^{4} P^{o}$ & 7.7071053 & 7.7063 & \\
\hline ......... & $1 s\left({ }^{2} S\right) 2 s 2 p\left({ }^{3} P^{o}\right){ }^{4} P_{3 / 2}^{o}$ & 7.7119406 & 7.7127 & \\
\hline $3 \ldots$ & $1 s\left({ }^{2} S\right) 2 s 2 p\left({ }^{3} P^{o}\right){ }^{4} P^{o}$ & & 7.7291 & \\
\hline ........ & $1 s\left({ }^{2} S\right) 2 s 2 p\left({ }^{3} P^{o}\right){ }^{2} P_{1 / 2}^{o}$ & 7.7504997 & 7.7508 & \\
\hline $3 \ldots$ & $1 s\left({ }^{2} S\right) 2 s 2 p\left({ }^{3} P^{o}\right){ }^{2} P_{3 / 2}^{o}$ & 7.7635181 & 7.7657 & \\
\hline $3 \ldots$. & $1 s\left({ }^{2} S\right) 2 p^{2}\left({ }^{3} P\right){ }^{4} P_{5 / 2}$ & 7.7907946 & 7.7923 & \\
\hline 3. & $1 s\left({ }^{2} S\right) 2 p^{2}\left({ }^{3} P\right){ }^{4} P_{3 / 2}$ & 7.7836035 & 7.7841 & \\
\hline $3 \ldots$ & $1 s\left({ }^{2} S\right) 2 p^{2}\left({ }^{3} P\right){ }^{4} P_{1 / 2}$ & 7.7718250 & 7.7711 & \\
\hline 3. & $1 s\left({ }^{2} S\right) 2 s 2 p\left({ }^{1} P^{o}\right){ }^{2} P_{3 / 2}^{o}$ & 7.7826116 & 7.7830 & \\
\hline $3 \ldots$ & $1 s\left({ }^{2} S\right) 2 s 2 p\left({ }^{1} P^{o}\right){ }^{2} P_{1 / 2}^{o}$ & 7.7791401 & 7.7807 & \\
\hline ....... & $1 s\left({ }^{2} S\right) 2 p^{2}\left({ }^{3} P\right){ }^{2} P_{1 / 2}$ & 7.8098881 & 7.8119 & \\
\hline $3 \ldots$. & $1 s\left({ }^{2} S\right) 2 p^{2}\left({ }^{3} P\right){ }^{2} P_{3 / 2}$ & 7.8348090 & 7.8370 & \\
\hline ........... & $1 s\left({ }^{2} S\right) 2 p^{2}\left({ }^{3} P\right){ }^{2} D_{3 / 2}$ & 7.8098881 & 7.8108 & \\
\hline $3 \ldots \ldots \ldots$ & $1 s\left({ }^{2} S\right) 2 p^{2}\left({ }^{3} P\right)^{2} D_{5 / 2}$ & 7.8193109 & 7.8219 & \\
\hline 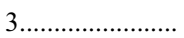 & $1 s\left({ }^{2} S\right) 2 p^{2}\left({ }^{3} P\right){ }^{2} S_{1 / 2}$ & 7.8548944 & 7.8572 & \\
\hline
\end{tabular}

${ }^{\text {a }}$ NIST database (Ralchenko et al. 2008). Level energies are determined by interpolation or extrapolation of known experimental values.

b HFR calculations (this work).

c $1 / Z$ expansion calculations (Vainshtein \& Safronova 1985).

and $\mathrm{K} \alpha_{2} / \mathrm{K} \alpha_{1}$ and $\mathrm{K} \beta / \mathrm{K} \alpha$ line ratios which can be compared to the available solid-state measurements (Salem \& Wimmer 1970; Salem et al. 1972; Slivinsky \& Ebert 1972; Berenyi et al. 1978; Rao et al. 1986; Perujo et al. 1987; Hölzer et al. 1997; Raj et al. 2001). The KLM/KLL and KMM/KLL Auger decay channel ratios are also investigated along the isonuclear sequence, and HFR relative Auger channel intensities in $\mathrm{Ni}^{+}$are compared with a recent experiment (Egri et al. 2008). Finally, the trends of the $\mathrm{K}$-shell fluorescence yield and natural K-level width with electron number $N$ are studied.

\subsection{Energy Levels}

$\mathrm{K}$-vacancy level energies for $\mathrm{Ni}$ ions are very scarce in the literature. The NIST database (Ralchenko et al. 2008) lists values for He- and Li-like $\mathrm{Ni}$ that are interpolated or extrapolated from experimental level energies along isoelectronic sequences. Vainshtein \& Safronova (1985) calculated values in $\mathrm{Ni}^{26+}$ using the $1 / Z$ expansion technique. In Table 1 , we compare our HFR level energies with the two above-mentioned data sets, finding an agreement within $3 \mathrm{eV}$.

\subsection{Wavelengths}

The only wavelength measurements for $\mathrm{Ni} \mathrm{K}$ lines available in the literature are those obtained for highly charged ions $(N=$ 2-5) in tokamak plasmas (Hsuan et al. 1987; Bombarda et al. 1988) and in the solid state with the recent experiment of Hölzer et al. (1997). Previous calculations are due to Vainshtein \& Safronova $(1978,1985)$ using the $1 / Z$ expansion technique for He- and Li-like Ni, Hsuan et al. (1987) by means of the HartreeFock-Slater (HFS) method to interpret observations in He- and Li-like Ni, and Bombarda et al. (1988) with SUPERSTRUCTURE to model measurements in $\mathrm{He}$ - to B-like Ni. 
TABLE 2

WaVelengths For K Lines in Ni Ions with $2 \leq N \leq 5$

\begin{tabular}{|c|c|c|c|c|c|}
\hline \multirow[b]{2}{*}{$N$} & \multirow[b]{2}{*}{ LOWER LEVEL } & \multirow[b]{2}{*}{ UPPER LEVEL } & \multicolumn{3}{|c|}{$\lambda(\AA)$} \\
\hline & & & $\mathrm{HFR}^{\mathrm{a}}$ & EXP & Other Theory \\
\hline $2 \ldots \ldots$ & $1 s^{2}{ }^{1} S_{0}$ & $1 s 2 p^{1} P_{1}^{o}$ & 1.5880 & $\begin{array}{l}1.5886^{\mathrm{b}} \\
1.5879^{\mathrm{c}} \\
1.5886^{\mathrm{d}}\end{array}$ & $\begin{array}{l}1.5856^{\mathrm{e}} \\
1.5879^{\mathrm{f}} \\
1.58848^{\mathrm{g}} \\
1.5886^{\mathrm{h}}\end{array}$ \\
\hline $2 \ldots \ldots \ldots \ldots \ldots \ldots \ldots \ldots \ldots$ & $1 s^{2}{ }^{1} S_{0}$ & $1 s 2 p^{3} P_{1}^{o}$ & 1.5963 & $\begin{array}{l}1.5966^{\mathrm{b}} \\
1.5962^{\mathrm{c}} \\
1.5969^{\mathrm{d}}\end{array}$ & $\begin{array}{l}1.5941^{\mathrm{e}} \\
1.5959^{\mathrm{f}} \\
1.59659^{\mathrm{g}} \\
1.5968^{\mathrm{h}}\end{array}$ \\
\hline 3 & $1 s^{2} 2 s^{2} S_{1 / 2}$ & $1 s\left({ }^{2} S\right) 2 s 2 p\left({ }^{1} P^{o}\right)^{2} P_{1 / 2}^{o}$ & 1.5935 & $\begin{array}{l}1.5940^{\mathrm{b}} \\
1.5931^{\mathrm{c}} \\
1.5938^{\mathrm{d}}\end{array}$ & $\begin{array}{l}1.5911^{\mathrm{e}} \\
1.5934^{\mathrm{f}} \\
1.5940^{\mathrm{h}}\end{array}$ \\
\hline 3 & $1 s^{2} 2 s^{2} S_{1 / 2}$ & $1 s\left({ }^{2} S\right) 2 s 2 p\left({ }^{3} P^{o}\right)^{2} P_{3 / 2}^{o}$ & 1.5966 & $\begin{array}{l}1.5972^{\mathrm{b}} \\
1.5962^{\mathrm{c}} \\
1.5969^{\mathrm{d}}\end{array}$ & $\begin{array}{l}1.5941^{\mathrm{e}} \\
1.5965^{\mathrm{f}} \\
1.5972^{\mathrm{h}}\end{array}$ \\
\hline $3 \ldots \ldots \ldots \ldots \ldots \ldots \ldots \ldots$ & $1 s^{2} 2 p^{2} P_{1 / 2}^{o}$ & $1 s\left({ }^{2} S\right) 2 p^{2}\left({ }^{1} D\right)^{2} D_{3 / 2}$ & 1.5981 & $\begin{array}{l}1.5987^{\mathrm{b}} \\
1.5978^{\mathrm{c}} \\
1.5985^{\mathrm{d}}\end{array}$ & $\begin{array}{l}1.5956^{\mathrm{e}} \\
1.5980^{\mathrm{f}} \\
1.5987^{\mathrm{h}}\end{array}$ \\
\hline 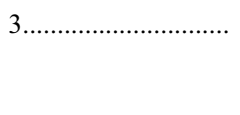 & $1 s^{2} 2 s^{2} S_{1 / 2}$ & $1 s\left({ }^{2} S\right) 2 s 2 p\left({ }^{3} P^{o}\right)^{2} P_{1 / 2}^{o}$ & 1.5996 & $\begin{array}{l}1.5999^{\mathrm{b}} \\
1.5991^{\mathrm{c}} \\
1.5998^{\mathrm{d}}\end{array}$ & $\begin{array}{l}1.5973^{\mathrm{e}} \\
1.5992^{\mathrm{f}} \\
1.6003^{\mathrm{h}}\end{array}$ \\
\hline 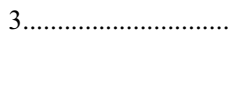 & $1 s^{2} 2 p^{2} P_{3 / 2}^{o}$ & $1 s\left({ }^{2} S\right) 2 p^{2}\left({ }^{1} D\right){ }^{2} D_{5 / 2}$ & 1.6004 & $\begin{array}{l}1.6011^{\mathrm{b}} \\
1.6003^{\mathrm{c}} \\
1.6010^{\mathrm{d}}\end{array}$ & $\begin{array}{l}1.5983^{\mathrm{e}} \\
1.6005^{\mathrm{f}} \\
1.6011^{\mathrm{h}}\end{array}$ \\
\hline $3 \ldots \ldots \ldots$ & $1 s^{2} 2 p^{2} P_{3 / 2}^{o}$ & $1 s\left({ }^{2} S\right) 2 p^{2}\left({ }^{1} D\right)^{2} D_{3 / 2}$ & 1.6027 & $\begin{array}{l}1.6029^{\mathrm{c}} \\
1.6036^{\mathrm{d}}\end{array}$ & $\begin{array}{l}1.6024^{\mathrm{f}} \\
1.6033^{\mathrm{h}}\end{array}$ \\
\hline $4 \ldots \ldots \ldots \ldots \ldots \ldots \ldots \ldots$ & $1 s^{2} 2 s^{2}{ }^{1} S_{0}$ & $1 s 2 s^{2} 2 p^{1} P_{1}^{o}$ & 1.6039 & $\begin{array}{l}1.6046^{\mathrm{b}} \\
1.6037^{\mathrm{c}} \\
1.6044^{\mathrm{d}}\end{array}$ & $\begin{array}{l}1.6015^{\mathrm{e}} \\
1.6047^{\mathrm{h}}\end{array}$ \\
\hline $4 \ldots \ldots \ldots \ldots \ldots \ldots \ldots \ldots$ & $1 s^{2} 2 s 2 p^{3} P_{2}^{o}$ & $1 s 2 s 2 p^{2}{ }^{3} D_{3}$ & 1.6082 & $1.6090^{\mathrm{b}}$ & $1.6064^{\mathrm{e}}$ \\
\hline 4 & $1 s^{2} 2 s 2 p{ }^{1} P_{1}^{o}$ & $1 s 2 s 2 p^{2}{ }^{1} D_{2}$ & 1.6106 & $1.6110^{\mathrm{b}}$ & $1.6087^{\mathrm{e}}$ \\
\hline 5 & $1 s^{2} 2 s^{2} 2 p^{2} P_{3 / 2}^{o}$ & $1 s 2 s^{2} 2 p^{2}{ }^{2} S_{1 / 2}$ & 1.6116 & $1.6123^{\mathrm{b}}$ & $1.6097^{\mathrm{e}}$ \\
\hline 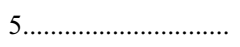 & $1 s^{2} 2 s^{2} 2 p^{2} P_{3 / 2}^{o}$ & $1 s 2 s^{2} 2 p^{2}{ }^{2} P_{3 / 2}$ & 1.6130 & $1.6135^{\mathrm{b}}$ & $1.6109^{\mathrm{e}}$ \\
\hline 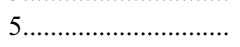 & $1 s^{2} 2 s^{2} 2 p^{2} P_{1 / 2}^{o}$ & $1 s 2 s^{2} 2 p^{2}{ }^{2} D_{3 / 2}$ & 1.6137 & $1.6144^{\mathrm{b}}$ & $1.6117^{\mathrm{e}}$ \\
\hline $5 \ldots \ldots \ldots \ldots \ldots$ & $1 s^{2} 2 s^{2} 2 p^{2} P_{1 / 2}^{o}$ & $1 s 2 s^{2} 2 p^{2}{ }^{2} P_{1 / 2}$ & 1.6138 & $1.6144^{\mathrm{b}}$ & $1.6118^{\mathrm{e}}$ \\
\hline $5 \ldots \ldots \ldots \ldots \ldots \ldots \ldots \ldots$ & $1 s^{2} 2 s^{2} 2 p^{2} P_{3 / 2}^{o}$ & $1 s 2 s^{2} 2 p^{2}{ }^{2} D_{5 / 2}$ & 1.6159 & $1.6167^{\mathrm{b}}$ & $1.6141^{\mathrm{e}}$ \\
\hline
\end{tabular}

${ }^{\text {a }}$ HFR calculations (this work).

b JET tokamak measurements by Bombarda et al. (1988). The absolute accuracy is approximately $\pm 0.001 \AA$.

c TFTR tokamak measurements by Hsuan et al. (1987). Normalized to the theoretical value of Vainshtein \& Safronova (1978) for the $1 s^{2}{ }^{1} S_{0}-1 s 2 p^{1} P_{1}$ He-like transition.

TFTR tokamak measurements by Hsuan et al. (1987). Normalized to the HFS value (Hsuan et al. 1987) for the $1 s^{2}{ }^{1} S_{0}-1 s 2 p{ }^{1} P_{1}$ He-like transition.

e SUPERSTRUCTURE calculations by Bombarda et al. (1988).

f $1 / Z$ expansion calculations by Vainshtein \& Safronova (1978).

g $1 / Z$ expansion calculations by Vainshtein \& Safronova (1985).

${ }^{\text {h }}$ HFS calculations by Hsuan et al. (1987).

These different sets of wavelengths are compared to our HFR calculation in Table 2 for ions with $N=2-5$. The agreement with experiment, the $1 / Z$ expansion, and the HFS calculations is within $1 \mathrm{~m} \AA$. Concerning the somewhat larger discrepancies encountered with the SUPERSTRUCTURE calculation of Bombarda et al. (1988), we believe that the discrepancies are due to their optimization procedure, since they optimized the atomic orbitals in the Li-like system and kept the orbitals fixed for all the other ions.

HFR centroid wavelengths in the second- and third-row ions $(N=12-27)$ are plotted as a function of $N$ for the $\mathrm{K} \alpha_{1,2}$ (Fig. 1) and $\mathrm{K} \beta$ (Fig. 2) UTAs. It may be seen that the $\mathrm{K} \alpha_{1,2}$ lines are sharply shifted to the red as $N$ increases up to $N=17$ and from then on slowly shifted to the blue. This was also the case in our previous studies of the Fe K lines (Palmeri et al. 2003a, 2003b) and is consistent with the trend calculated by House (1969). The
$\mathrm{K} \beta$ line, in contrast, is monotonically shifted to the red with an increasing $N$. The values measured by Hölzer et al. (1997) for the $\mathrm{K} \alpha_{1}, \mathrm{~K} \alpha_{2}$, and $\mathrm{K} \beta$ solid-state UTAs are also shown in both figures, namely $\lambda \lambda 1.65790(1), 1.66175(1)$, and 1.500152(3), respectively. They compare favorably with our HFR values for $\mathrm{Ni}^{+}(N=27): \lambda \lambda 1.6575,1.6614,1.5000$. As for the highly charged ions, the accord is better than $1 \mathrm{~m} \AA$.

\subsection{A-Values, Radiative Widths, and Line Ratios}

A comparison of our HFR $A$-values for $\mathrm{K}$ transitions in ions with $3 \leq N \leq 5$ with the data of Bombarda et al. (1988) and Vainshtein \& Safronova (1978) is given in Figure 3, where the ratios with respect to HFR are plotted as a function of the HFR $A$-value. A large scatter is observed for $A \lesssim 10^{13} \mathrm{~s}^{-1}$, especially with the $A$-values for the Li-like system by Vainshtein \& 


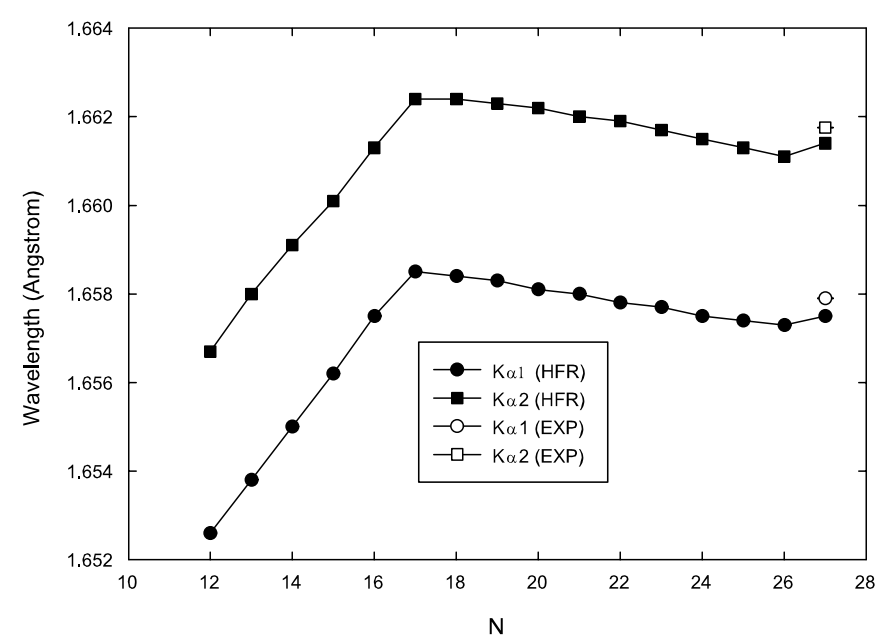

FIG. 1.-HFR wavelengths (in angstroms) for the $\mathrm{K} \alpha_{1}$ ( filled circles) and $\mathrm{K} \alpha_{2}$ (filled squares) UTAs in Ni ions with electron number $12 \leq N \leq 27$. The solid-state measurements by Hölzer et al. (1997) are also shown; open circle: $\mathrm{K} \alpha_{1}$; open square: $\mathrm{K} \alpha_{2}$.

Safronova (1978), thus illustrating the model dependency of the weak rates. The average ratios in the Li-like species are $0.98 \pm 0.07$ for Bombarda et al. (1988) and $0.91 \pm 0.31$ for Vainshtein \& Safronova (1978). The 31\% standard deviation observed in the latter is due to the weaker rates which are not listed by Bombarda et al. (1988). Concerning the Be- and B-like ions, where the only available data are given in Bombarda et al. (1988), the average ratios are, respectively, $1.02 \pm 0.08$ and $1.04 \pm 0.01$. Based on these comparisons, we are confident that our $A$-values have a $10 \%$ accuracy for transitions with $A \gtrsim 10^{13} \mathrm{~s}^{-1}$.

In Figure 4 we compare our HFR radiative widths with the values obtained by Bombarda et al. (1988) for systems with $3 \leq$ $N \leq 5$. The ratio with respect to HFR is plotted as a function of the HFR width. If the $1 s 2 s^{2} 2 p^{2}{ }^{4} P_{1 / 2}$ level in the B-like ion is excluded from this comparison, the average ratios are then $0.99 \pm$ 0.07 for $N=3,1.01 \pm 0.04$ for $N=4$, and $1.04 \pm 0.03$ for $N=5$. Concerning the excluded level, the width of $6.86 \times 10^{14} \mathrm{~s}^{-1}$ quoted by Bombarda et al. (1988) is questionable. It differs by 1 order of magnitude with our HFR width $\left(6.20 \times 10^{13} \mathrm{~s}^{-1}\right)$ and with the value they list for the other level of the same multiplet,

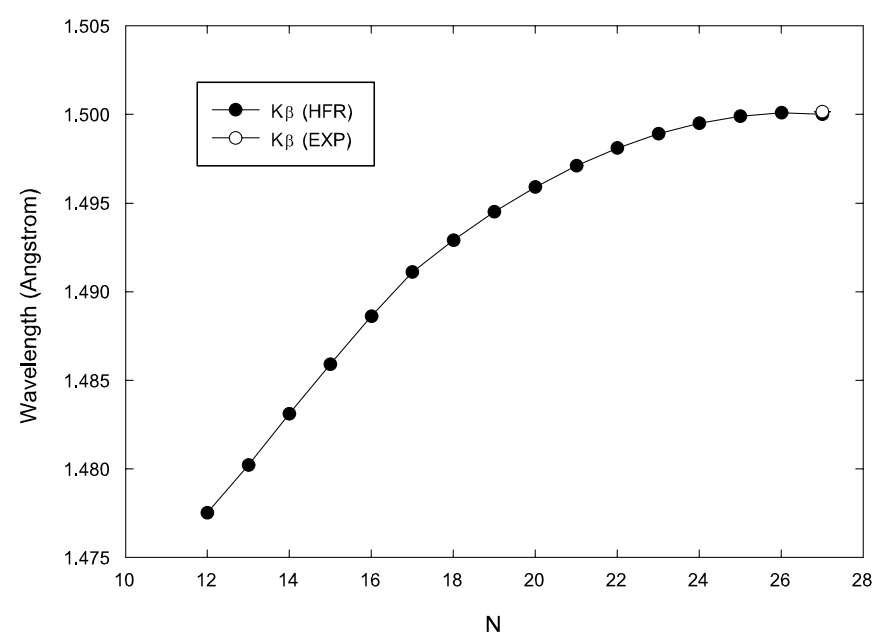

FIG. 2.-HFR wavelengths (in angstroms) of the $\mathrm{K} \beta \mathrm{UTA}$ for Ni ions with electron number $12 \leq N \leq 27$ ( filled circles). The solid-state measurement by Hölzer et al. (1997) is also shown (open circle).
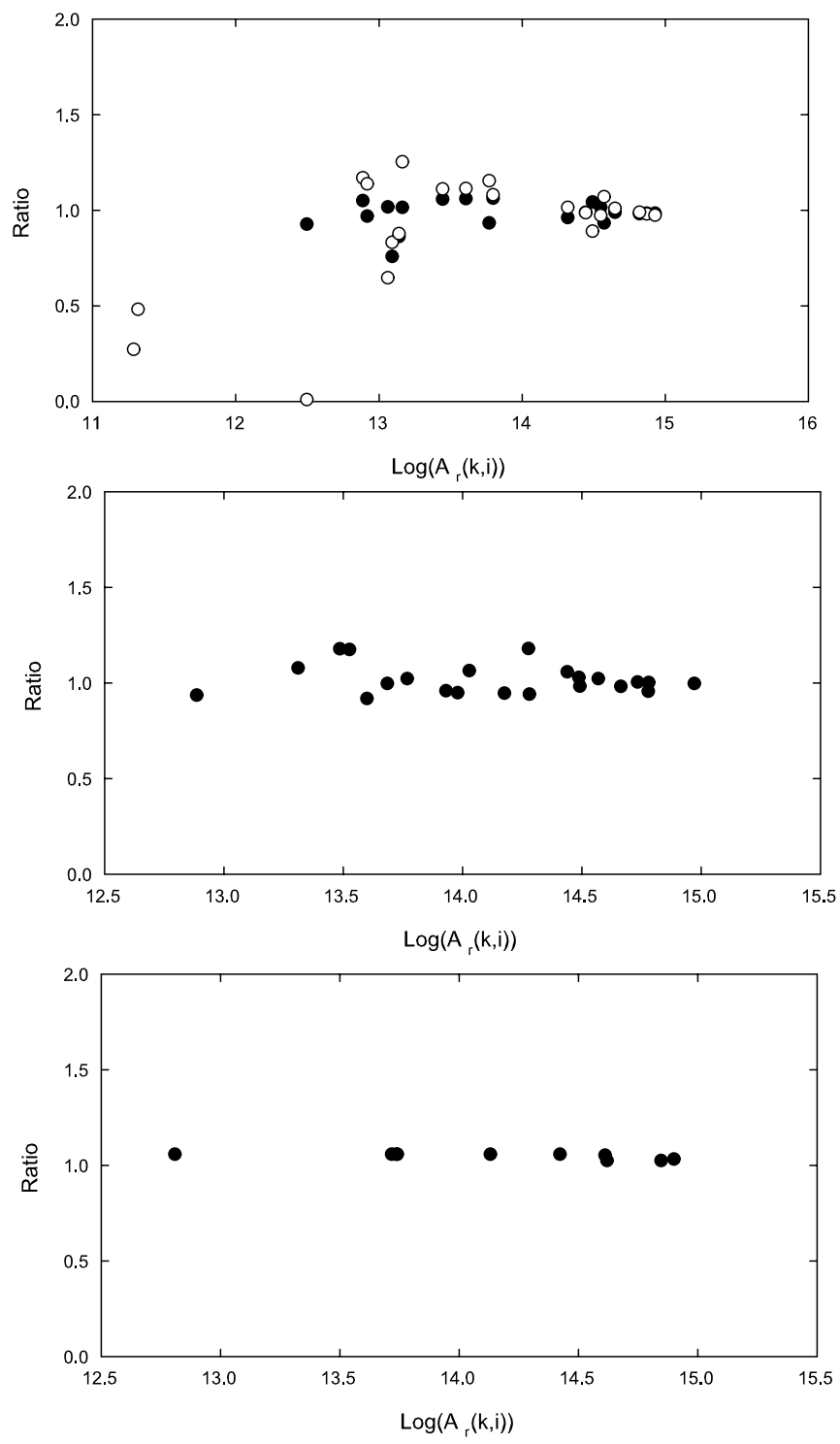

FIG. 3.-Comparison of the present HFR $A$-values for $\mathrm{K}$ transitions in Li-like (top), Be-like (middle), and B-like nickel (bottom) with two independent calculations. Filled circles: Bombarda et al. (1988). Open circles: Vainshtein \& Safronova (1978).

namely $6.35 \times 10^{13} \mathrm{~s}^{-1}$ for $1 s 2 s^{2} 2 p^{2}{ }^{4} P_{5 / 2}$. Thus, this error must be due to a misprint and the agreement is better than $10 \%$.

The trend of the $\mathrm{K} \alpha_{2} / \mathrm{K} \alpha_{1}$ line ratio along the isonuclear sequence is shown in Figure 5 for the $\mathrm{K} \alpha_{1,2}$ UTAs in members with $12 \leq N \leq 27$. This line ratio increases with $N$ up to $N=15$, and then decreases to become almost constant at a value of $\sim 0.5$ for $N \geq 17$. This trend is consistent (within $10 \%$ ) in the three numerical approaches that we considered, i.e., HFR, AUTOSTRUCTURE, and MCDF. Moreover, our calculated line ratios for $N=27$ are compared with the Dirac-Fock value of Scofield (1974), and the two solid-state measurements of Salem \& Wimmer (1970) and Hölzer et al. (1997). All these results agree to within 5\%.

The contribution of satellites to the $\mathrm{K} \alpha_{2} / \mathrm{K}_{1}$ line ratio in $\mathrm{Ni}^{3+}$ is illustrated in Figure 6. Stick spectra, i.e., $A$-values as function of the transition wavelength, computed with HFR are plotted in the region of the $\mathrm{K} \alpha$ line for the diagram lines $\left([1 s] 3 d^{M}-[2 p] 3 d^{M}\right)$ in thin gray sticks and for the satellites $\left([1 s] 3 d^{M}-[2 p] 3 d^{M-1} 4 s\right.$ and $\left.[1 s] 3 d^{M}-[2 p] 3 d^{M-2} 4 s^{2}\right)$ in thick black sticks. The top panel 

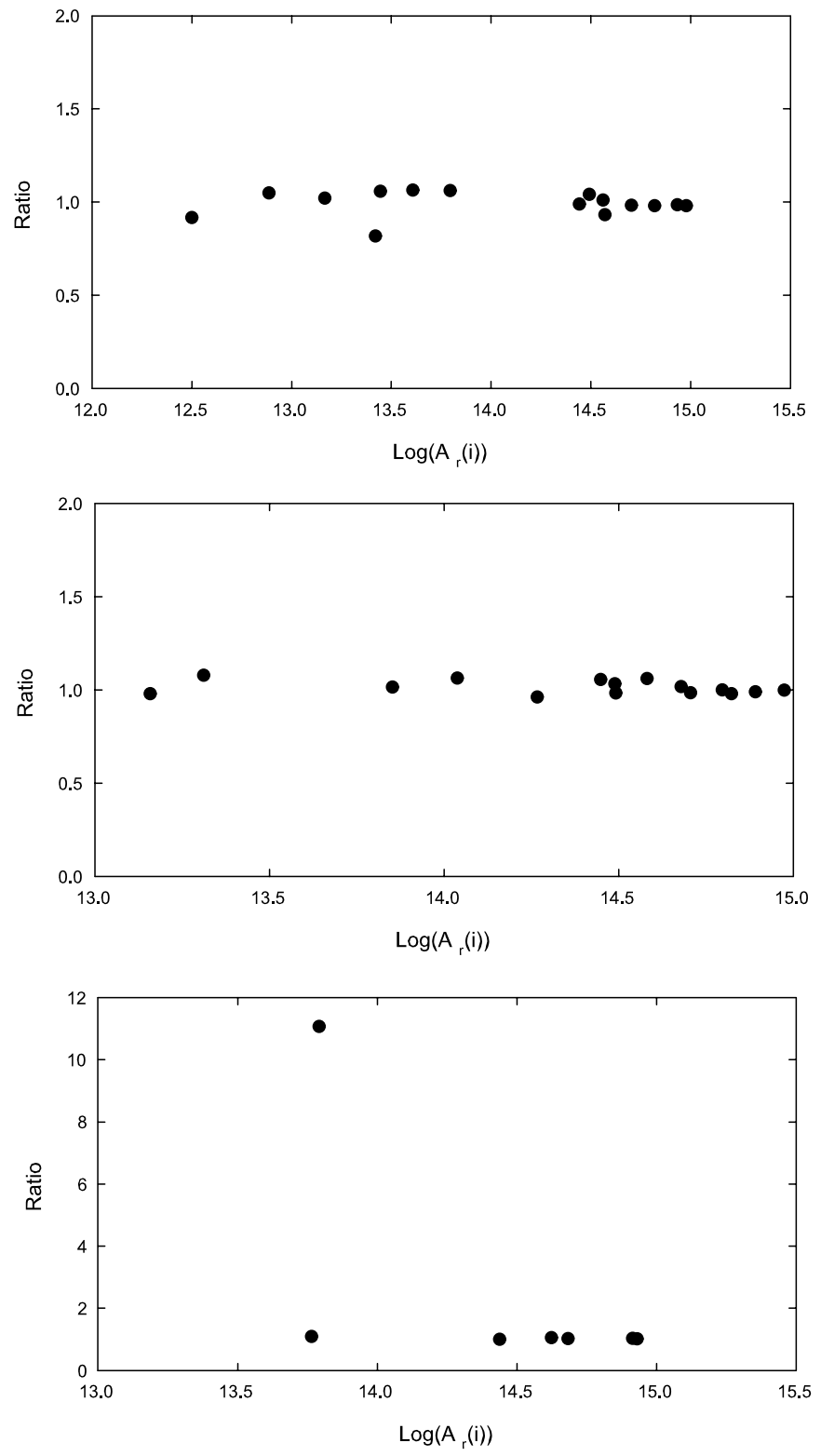

FIG. 4.-Same as Fig. 3, but for comparison of the present HFR radiative widths for K-vacancy levels.

shows the $\mathrm{Ni}^{2+}$ spectrum, the middle shows $\mathrm{Ni}^{3+}$, and the bottom shows $\mathrm{Ni}^{4+}$. One clearly sees that the satellites in $\mathrm{Ni}^{3+}$ are intense relative to the $\mathrm{K} \alpha_{2}$ diagram lines and are blended with the latter. As a consequence, the $\mathrm{K} \alpha_{2} / \mathrm{K} \alpha_{1}$ line ratio drops to 0.37 without satellites instead of having a value of 0.50 when included. In the other ions, their effect on the line ratio can be neglected.

The $\mathrm{K} \beta / \mathrm{K} \alpha$ line ratio is plotted as function of $N$ in Figure 7 for the $\mathrm{K} \alpha$ and $\mathrm{K} \beta$ UTAs in ions with $12 \leq N \leq 27$. One can see that the trend along the isonuclear sequence presents a sharp increase with $N$ up to $N=17$ due to the filling of the $3 p$ subshell, the fertile domain of the $\mathrm{K} \beta$ transition, followed by a slow decrease associated with the filling of the $3 d$ subshell. This behavior is confirmed with our three independent numerical methods (HFR, AUTOSTRUCTURE, and MCDF) to within 10\%.

In Figure 7 our computed $\mathrm{K} \beta / \mathrm{K} \alpha$ line ratios for $N=27$ are compared with the solid-state measurements (Salem et al. 1972; Slivinsky \& Ebert 1972; Berenyi et al. 1978; Rao et al. 1986; Perujo et al. 1987; Hölzer et al. 1997; Raj et al. 2001) and other theoretical values (Scofield 1974; Polasik 1998). The bulk of the experimental

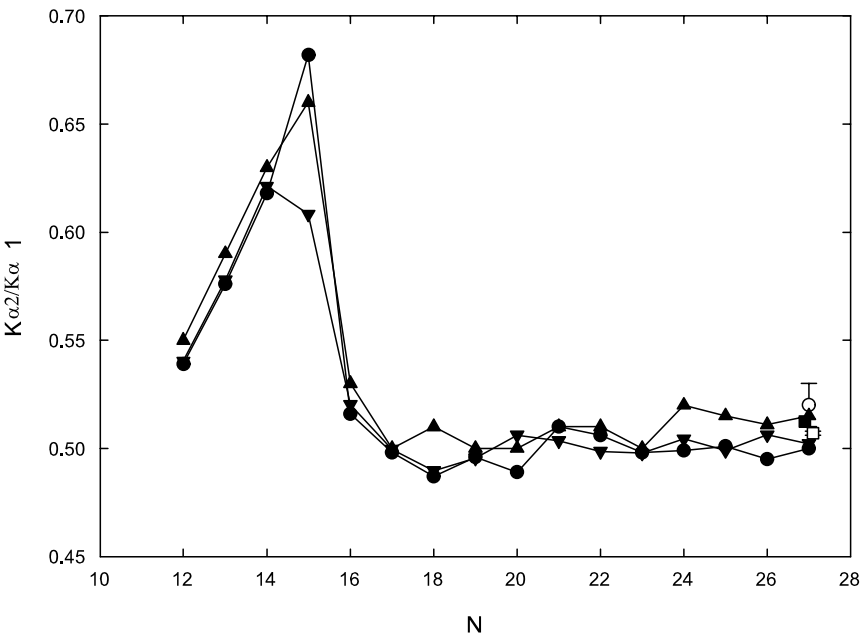

FIg. 5.-The $\mathrm{K} \alpha_{2} / \mathrm{K} \alpha_{1}$ line ratio for $\mathrm{Ni}$ ions with electron number $12 \leq N \leq$ 27. Filled circles: HFR (this work). Filled upright triangles: MCDF-EAL (this work). Filled inverted triangles: AUTOSTRUCTURE (this work). Filled squares: Dirac-Fock calculation (Scofield 1974). Open circle: Solid-state measurement by Hölzer et al. (1997). Open square: Measurement in the solid by Salem \& Wimmer (1970).
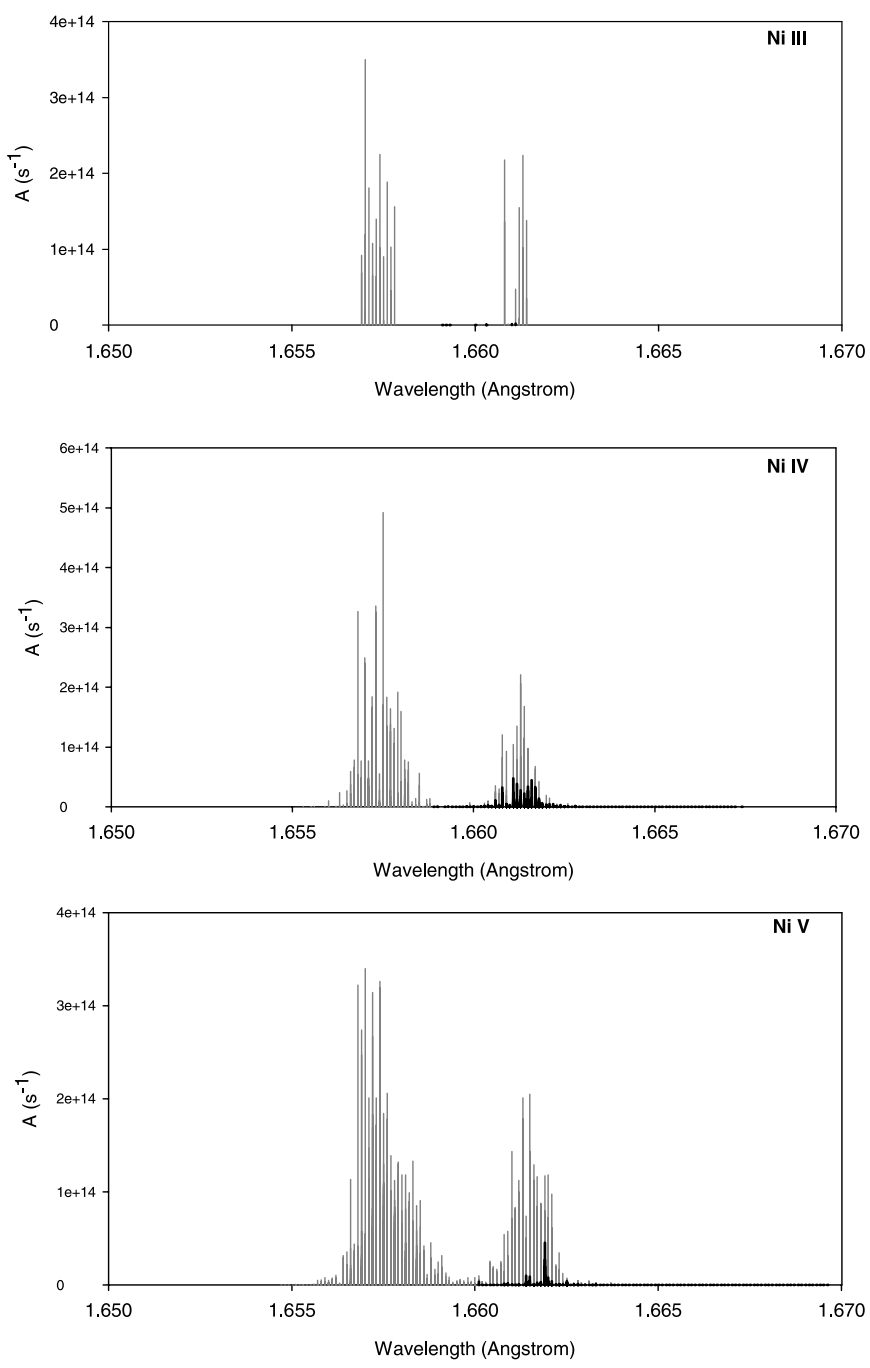

FIG. 6.-Stick spectra, i.e., $A$-value ( $\mathrm{s}^{-1}$ ) vs. wavelength (in angstroms), in the region of the $\mathrm{Ni} \mathrm{K} \alpha$ line computed with HFR in Ni III (top), Ni Iv (middle), and Ni v (bottom). Thin gray sticks are diagram lines, $[1 s] 3 d^{M}-[2 p] 3 d^{M}$, while the thick black sticks are the $[1 s] 3 d^{M}-[2 p] 3 d^{M-1} 4 s$ and $[1 s] 3 d^{M}-[2 p] 3 d^{M-2} 4 s^{2}$ satellite lines. 


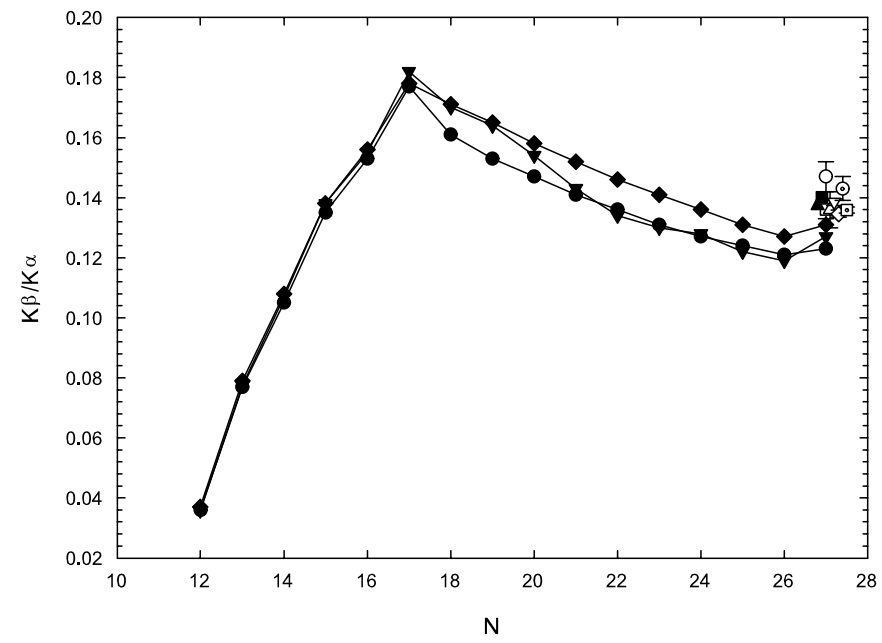

Fig. 7.- The $\mathrm{K} \beta / \mathrm{K} \alpha$ line ratio for $\mathrm{Ni}$ ions with electron number $12 \leq N \leq 27$. Filled circles: HFR (this work). Filled inverted triangles: AUTOSTRUCTURE (this work). Filled diamonds: MCDF-EAL (this work). Filled square: Dirac-Fock calculation (Scofield 1974). Filled upright triangle: MCDF-SAL calculation (Polasik 1998). The solid-state measurements are as follows. Open circle: Hölzer et al. (1997). Open square: Salem et al. (1972). Open upright triangle: Rao et al. (1986). Open inverted triangle: Slivinsky \& Ebert (1972). Open diamond: Raj et al. (2001). Dotted circle: Berenyi et al. (1978). Dotted square: Perujo et al. (1987).

values are located at $0.139 \pm 0.015$ (this deviation takes into account the experimental error bars), which can be compared to the ratios we computed with HFR (0.117), AUTOSTRUCTURE (0.126), and MCDF (0.131). Other theoretical values are also close to experiment, namely the Dirac-Fock result by Scofield (1974) at 0.140 and that with the MCDF-SAL method of 0.137 (Polasik 1998). Therefore, there is good theory-experiment agreement in $\mathrm{Ni}^{+}$except for our HFR ratio, which is $15 \%$ lower.

\subsection{Auger Widths and Auger Channel Ratios}

For ions with electron number $3 \leq N \leq 5$, we compare HFR Auger widths in Figure 8 with the results by Bombarda et al. (1988) and Vainshtein \& Safronova (1978). Ratios with respect to HFR are plotted as a function of the HFR Auger width. Large scatter may be observed for $A_{a} \lesssim 10^{13} \mathrm{~s}^{-1}$. Excluded from this figure are the ratios for the $1 s\left({ }^{2} S\right) 2 s 2 p\left({ }^{3} P^{o}\right)^{2} P_{3 / 2}^{o}$ level in the Li-like system which has a small width, namely $A_{a}(\mathrm{HFR})=6 \times$ $10^{9} \mathrm{~s}^{-1}$, for which large discrepancies are found. If ratios less than $10^{13} \mathrm{~s}^{-1}$ are discarded, the average ratios in $\mathrm{Ni}^{25+}$ are $1.04 \pm 0.03$ (Bombarda et al. 1988) and $1.14 \pm 0.10$ (Vainshtein \& Safronova 1978). The accord between these three independent methods is fair, although the widths obtained by the latter are significantly higher ( $\sim 15 \%$ on average) than HFR. Concerning the other two ions, where the only available data are those by Bombarda et al. (1988), the average ratios are $1.10 \pm 0.05\left(\mathrm{Ni}^{24+}\right)$ and $1.15 \pm 0.03\left(\mathrm{Ni}^{23+}\right)$. These systematic deviations (10\% and $15 \%$ higher than HFR, respectively, in the Be- and B-like species) may be due, as previously mentioned, to the fact that Bombarda et al. (1988) used orbitals optimized in the Li-like system.

The trends of the HFR KLM/KLL and KMM/KLL channel ratios along the isonuclear sequence $(17 \leq N \leq 27)$ are depicted in Figures 9 and 10, respectively, and are found to be similar to those previously observed in iron (Palmeri et al. 2003b). Unfortunately, here there is no available solid-state measurements to compare with the theoretical ratios.

In Table 3 the HFR KLM Auger channel relative intensities in $\mathrm{Ni}^{+}$are compared with the recent measurements of Egri et al.
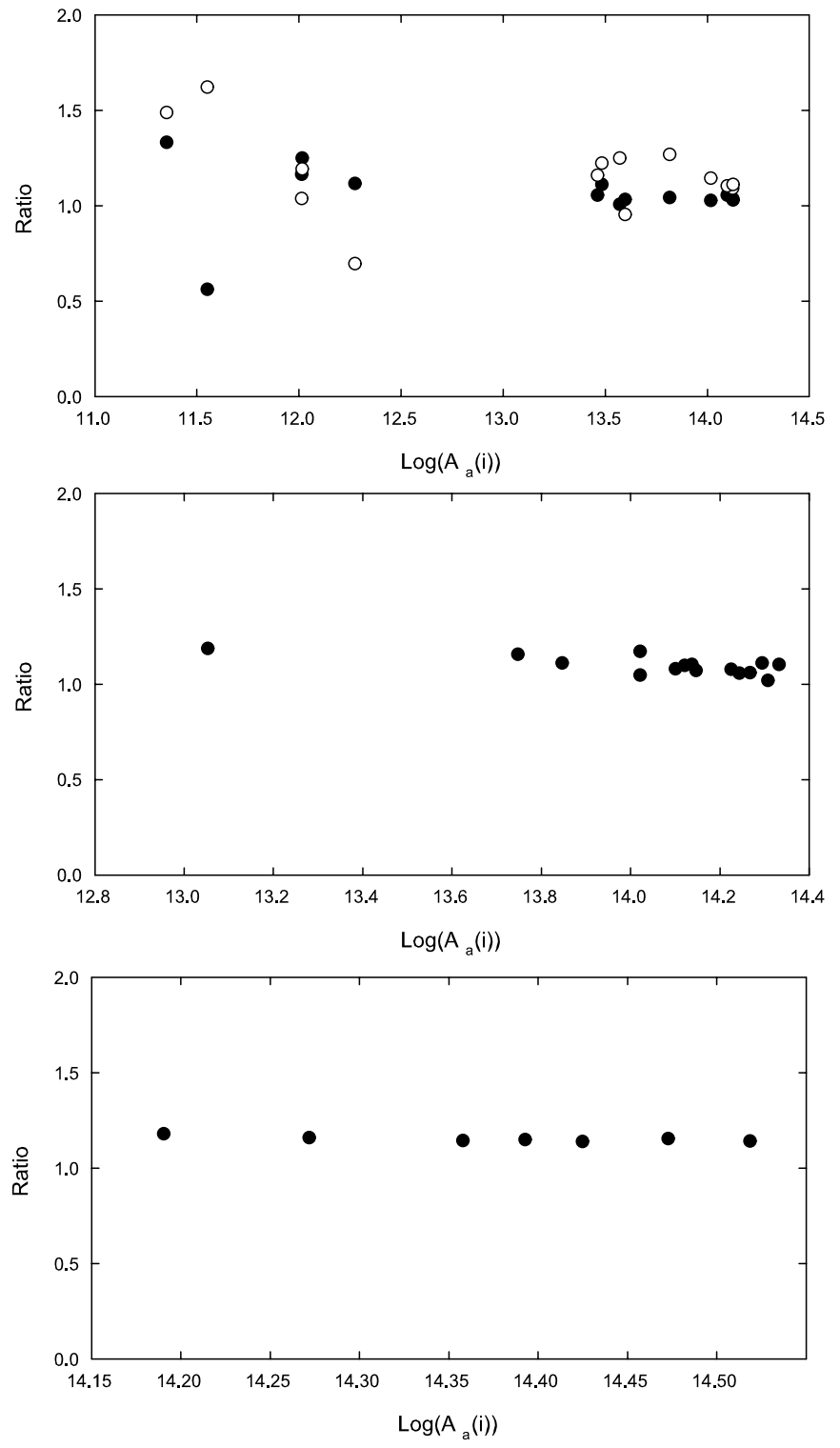

FIG. 8. - Comparison of the present HFR Auger widths for K-vacancy levels in Li-like (top), Be-like (middle), and B-like nickel (bottom) with two independent calculations. Filled circles: Bombarda et al. (1988). Open circles: Vainshtein \& Safronova (1978).

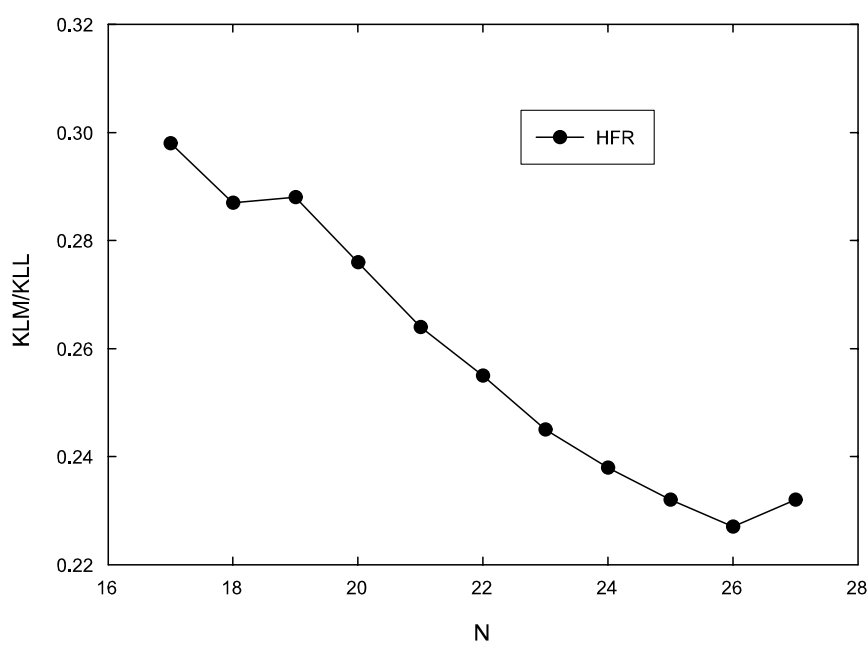

FIG. 9.-HFR KLM/KLL Auger channel ratio for Ni ions with electron number $17 \leq N \leq 27$ 


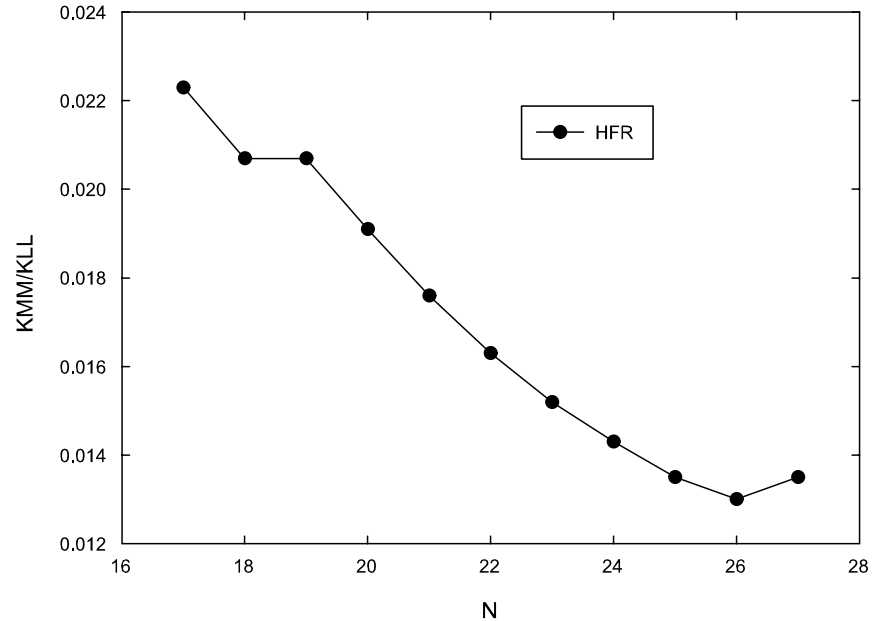

FIG. 10.-HFR KMM/KLL Auger channel ratio for $\mathrm{Ni}$ ions with electron number $17 \leq N \leq 27$.

(2008) made in the solid. Both data sets agree within the experimental error $(\sim 20 \%)$.

\subsection{Fluorescence Yields and Natural K-Level Widths}

The HFR average K-shell fluorescence yield, $\bar{\omega}_{K}$, for Ni ions with $3 \leq N \leq 27$ is plotted in Figure 11 as a function of $N$. For each ion, the average is computed over all fine-structure levels,

$$
\bar{\omega}_{K}=\frac{1}{m} \sum_{i=1}^{m} \omega_{K}(i)
$$

where

$$
\omega_{K}(i)=\frac{A_{r}(i)}{A_{a}(i)+A_{r}(i)}
$$

is the level yield. The following behavior may be noted: $\bar{\omega}_{K}$ sharply decreases from $0.80(N=3)$ to $0.46(N=9)$ in the firstrow ions, then slowly decreases from $0.46(N=10)$ to 0.43 $(N=17)$ in the second-row ions, and becomes constant in the third-row ions at a value of 0.43 for $\mathrm{Ni}^{+}(N=27)$. The recommended value of Hubbell et al. (1994), $0.412 \pm 0.012$, is also shown while our HFR value for $N=27$ is slightly higher by less than $5 \%$.

In Figure 12 the HFR natural K-level width, $\Gamma_{K}$ (in electron volts), is plotted along the nickel isonuclear sequence for ions with $3 \leq N \leq 27$. As for the fluorescence yields, the average for each ion is given by

$$
\bar{\Gamma}_{K}=\frac{1}{m} \sum_{i=1}^{m} \Gamma_{K}(i)
$$

\begin{tabular}{|c|c|c|c|}
\hline Ratio $^{\mathrm{a}}$ & $\mathrm{EXP}^{\mathrm{b}}$ & $\mathrm{HFR}^{\mathrm{c}}$ & HFR/EXP \\
\hline $\mathrm{KL}_{1} \mathrm{M}_{1} / \mathrm{KLM}$. & 0.076 & 0.086 & 1.13 \\
\hline $\mathrm{KL}_{1} \mathrm{M}_{23} / \mathrm{KLM} \ldots \ldots \ldots \ldots$ & 0.152 & 0.135 & 0.89 \\
\hline $\mathrm{KL}_{23} \mathrm{M}_{1} / \mathrm{KLM} \ldots \ldots \ldots \ldots \ldots \ldots \ldots$ & 0.136 & 0.123 & 0.90 \\
\hline $\mathrm{KL}_{23} \mathrm{M}_{23} / \mathrm{KLM}$ & 0.534 & 0.653 & 1.22 \\
\hline
\end{tabular}

\section{TABLE 3}

KLM Auger Channel Relative Intensities in $\mathrm{Ni}^{+}$

a The ratios are relative to the total KLM Auger rate. $\mathrm{K}=1 s ; \mathrm{L}_{1}=2 s ; \mathrm{L}_{23}=$ $2 p ; \mathrm{M}_{1}=3 s ; \mathrm{M}_{23}=3 p$.

b Solid-state measurements by Egri et al. (2008). The errors are about $20 \%$.

${ }^{\mathrm{c}}$ HFR calculations (this work).

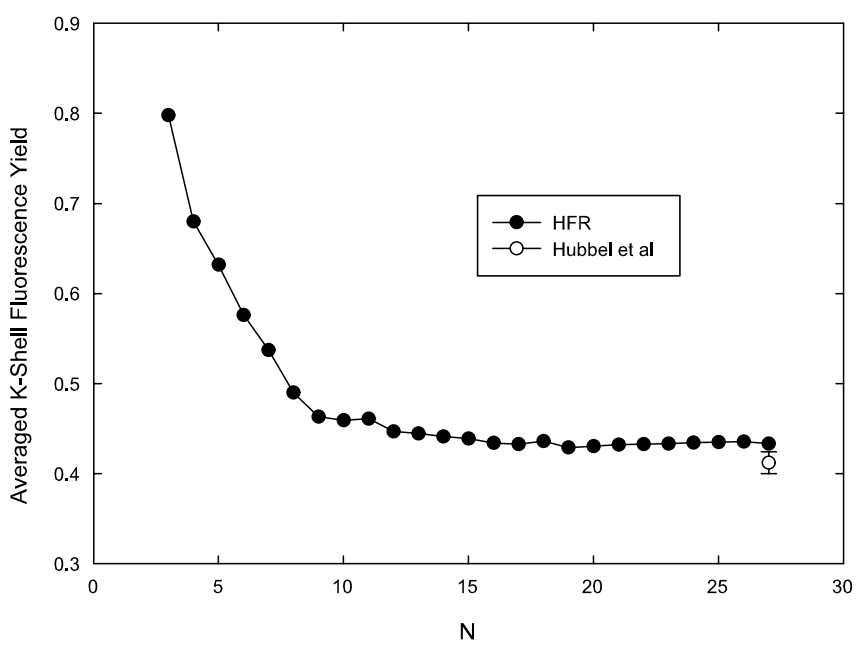

FIG. 11.-HFR averaged K-shell fluorescence yield for Ni ions with electron number $3 \leq N \leq 27$. The recommended value of Hubbell et al. (1994) is also shown.

where

$$
\Gamma_{K}(i)=A_{a}(i)+A_{r}(i)
$$

and the standard deviation

$$
\sigma\left(\Gamma_{K}\right)=\sqrt{\frac{1}{m} \sum_{i=1}^{m}\left[\Gamma_{K}(i)-\bar{\Gamma}_{K}\right]^{2}} .
$$

The value of $\bar{\Gamma}_{K}$ increases sharply from $0.20 \mathrm{eV}$ for $N=3$ to $1.16 \mathrm{eV}$ for $N=9$, delimiting the first-row regime; then, in the second-row regime, it slowly increases from $1.21 \mathrm{eV}$ for $N=10$ to $1.41 \mathrm{eV}$ for $N=17$; and finally, it slowly decreases from $1.40 \mathrm{eV}$ for $N=18$ to $1.33 \mathrm{eV}$ for $N=27$ in the third-row regime. This last value agrees within $5 \%$ with the recommended natural K-level width of $1.39 \mathrm{eV}$ (Campbell \& Papp 2001), which is also plotted in the figure. In the third-row regime, the natural K-level width of each fine-structure level, $\Gamma_{K}(i)$, becomes constant in each ion, and thus, $\sigma\left(\Gamma_{K}\right)$ is negligible, as shown in the figure.

In Table 4, the natural K-level widths recommended by Campbell \& Papp (2001) for the singly ionized cosmically abundant elements

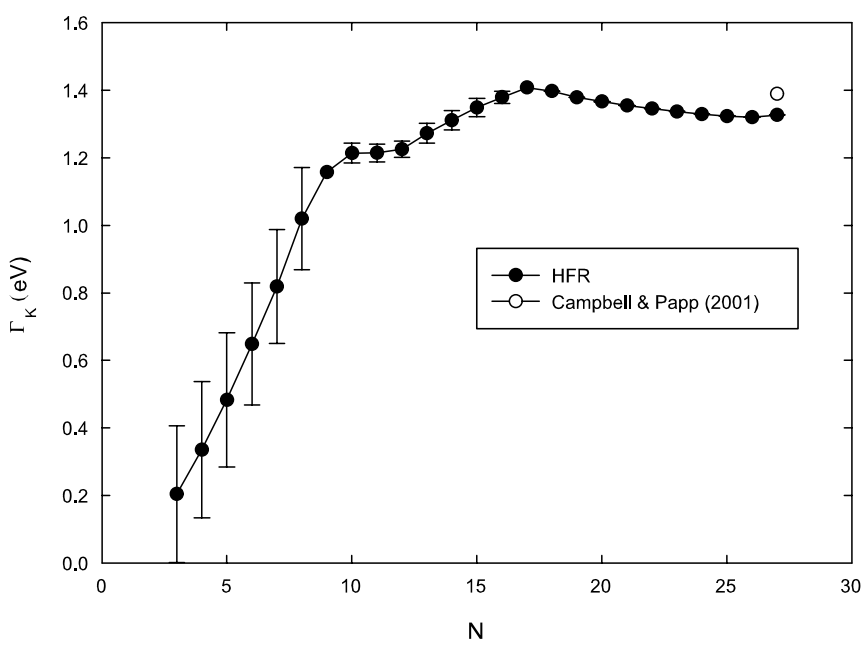

FIG. 12.-HFR natural K-level width $\Gamma_{K}$ (in electron volts) for Ni ions with electron number $3 \leq N \leq 27$. The average and standard deviation are plotted, the latter as error bars. The recommended value of Campbell \& Papp (2001) is also shown. 
TABLE 4

Natural $K$-Level Widths in Singly Ionized Cosmically Abundant Elements

\begin{tabular}{|c|c|c|}
\hline Element & $\begin{array}{c}\mathrm{REC}^{\mathrm{a}} \\
(\mathrm{eV})\end{array}$ & $\begin{array}{l}\text { HFR } \\
(\mathrm{eV})\end{array}$ \\
\hline $\mathrm{O} \ldots \ldots \ldots \ldots$ & 0.133 & $0.129^{\mathrm{b}}$ \\
\hline $\mathrm{Ne}$ & 0.24 & $0.247^{\mathrm{c}}$ \\
\hline $\mathrm{Mg}$ & 0.334 & $0.323^{\mathrm{c}}$ \\
\hline $\mathrm{Si}$ & 0.425 & $0.407^{\mathrm{c}}$ \\
\hline $\mathrm{S}$ & 0.552 & $0.532^{\mathrm{c}}$ \\
\hline Ar & 0.66 & $0.643^{\mathrm{c}}$ \\
\hline $\mathrm{Ca}$ & 0.77 & $0.756^{\mathrm{c}}$ \\
\hline Fe & 1.19 & $1.14^{\mathrm{d}}$ \\
\hline $\mathrm{Ni}$ & 1.39 & $1.33^{\mathrm{e}}$ \\
\hline $\begin{array}{l}\text { a } \text { Recommended value by } \\
\text { b } \text { HFR, García et al. (2005) } \\
\text { c HFR, Palmeri et al. (2008 } \\
\text { c HFR, Palmeri et al. (2003 } \\
\text { e HFR, this work. }\end{array}$ & bell \& & (2001). \\
\hline
\end{tabular}

are compared with HFR values calculated in the present work and in our earlier analyses (Palmeri et al. 2003b; García et al. 2005; Palmeri et al. 2008). The trend with $Z$ is well reproduced and both data sets agree satisfactorily.

\section{SUPPLEMENTARY ELECTRONIC TABLES}

Computed level energies, wavelengths, radiative transition probabilities, absorption oscillator strengths, radiative and Auger widths, and $\mathrm{K}$-shell fluorescence yields in $\mathrm{Ni}^{+}-\mathrm{Ni}^{26+}$ can be accessed from Tables 5 and 6 in the electronic version of
TABLE 6

K-Vacancy Transitions in Ni Ions with $2 \leq N \leq 3$

\begin{tabular}{|c|c|c|c|c|c|c|}
\hline$N$ & $k$ & $i$ & $\begin{array}{c}\lambda \\
(\AA)\end{array}$ & $\begin{array}{c}A_{r}(k, i) \\
\left(\mathrm{s}^{-1}\right)\end{array}$ & $g f(i, k)$ & $\mathrm{CF}$ \\
\hline $2 \ldots \ldots \ldots \ldots \ldots$ & 7 & 1 & 1.5880 & $6.49 \mathrm{E}+14$ & $7.36 \mathrm{E}-01$ & -1.00 \\
\hline $2 \ldots \ldots$ & 5 & 1 & 1.5963 & $6.82 \mathrm{E}+13$ & $7.82 \mathrm{E}-02$ & -1.00 \\
\hline 3............... & 19 & 2 & 1.5886 & $8.27 \mathrm{E}+12$ & $6.25 \mathrm{E}-03$ & 0.03 \\
\hline 3.............. & 18 & 2 & 1.5927 & $1.15 \mathrm{E}+13$ & $1.75 \mathrm{E}-02$ & 0.03 \\
\hline 3.............. & 13 & 1 & 1.5928 & $3.13 \mathrm{E}+12$ & $4.76 \mathrm{E}-03$ & 0.01 \\
\hline 3.................. & 19 & 3 & 1.5932 & $3.55 \mathrm{E}+14$ & $2.70 \mathrm{E}-01$ & 0.92 \\
\hline 3.................. & 11 & 1 & 1.5935 & $3.11 \mathrm{E}+14$ & $2.37 \mathrm{E}-01$ & -0.46 \\
\hline 3 & 9 & 1 & 1.5966 & $6.61 \mathrm{E}+14$ & $1.01 \mathrm{E}+00$ & -0.95 \\
\hline 3.………... & 18 & 3 & 1.5973 & $8.49 \mathrm{E}+14$ & $1.30 \mathrm{E}+00$ & 0.97 \\
\hline 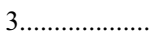 & 16 & 2 & 1.5979 & $7.44 \mathrm{E}+14$ & $5.69 \mathrm{E}-01$ & 0.97 \\
\hline $3 \ldots \ldots$ & 15 & 2 & 1.5981 & $4.46 \mathrm{E}+14$ & $6.84 \mathrm{E}-01$ & 0.95 \\
\hline 3..................... & 8 & 1 & 1.5996 & $3.73 \mathrm{E}+14$ & $2.86 \mathrm{E}-01$ & -0.94 \\
\hline 3.………........ & 17 & 3 & 1.6004 & $2.77 \mathrm{E}+14$ & $6.38 \mathrm{E}-01$ & 0.95 \\
\hline 3................... & 16 & 3 & 1.6025 & $2.09 \mathrm{E}+14$ & $1.61 \mathrm{E}-01$ & -0.47 \\
\hline 3........................... & 15 & 3 & 1.6027 & $5.88 \mathrm{E}+13$ & $9.06 \mathrm{E}-02$ & 0.13 \\
\hline 3..................... & 12 & 2 & 1.6039 & $1.94 \mathrm{E}+11$ & $2.99 \mathrm{E}-04$ & -0.02 \\
\hline 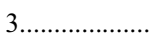 & 10 & 2 & 1.6064 & $4.04 \mathrm{E}+13$ & $3.13 \mathrm{E}-02$ & 0.94 \\
\hline 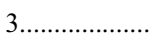 & 14 & 3 & 1.6066 & $6.26 \mathrm{E}+13$ & $1.45 \mathrm{E}-01$ & -0.95 \\
\hline 3...……… & 6 & 1 & 1.6075 & $2.79 \mathrm{E}+13$ & $4.33 \mathrm{E}-02$ & -0.96 \\
\hline $3 \ldots \ldots \ldots$ & 12 & 3 & 1.6085 & $1.46 \mathrm{E}+13$ & $2.26 \mathrm{E}-02$ & 0.97 \\
\hline 3..................... & 5 & 1 & 1.6089 & $7.72 \mathrm{E}+12$ & $6.00 \mathrm{E}-03$ & -0.96 \\
\hline 3..................... & 10 & 3 & 1.6110 & $2.08 \mathrm{E}+11$ & $1.61 \mathrm{E}-04$ & 0.01 \\
\hline 3..................... & 4 & 2 & 1.6225 & $1.38 \mathrm{E}+13$ & $1.09 \mathrm{E}-02$ & 0.76 \\
\hline 3.………........ & 4 & 3 & 1.6272 & $1.24 \mathrm{E}+13$ & $9.86 \mathrm{E}-03$ & 0.42 \\
\hline
\end{tabular}

Notes.-Table 6 is published in its entirety in the electronic edition of the Astrophysical Journal Supplement. A portion is shown here for guidance regarding its form and content.

TABLE 5

Ni Valence and K-Vacancy Levels with $2 \leq N \leq 3$

\begin{tabular}{|c|c|c|c|c|c|c|c|c|c|}
\hline$N$ & $i$ & $2 S+1$ & $L$ & $2 J$ & Conf & $\begin{array}{c}E \\
(\mathrm{keV})\end{array}$ & $\begin{array}{l}A_{r}(i) \\
\left(\mathrm{s}^{-1}\right)\end{array}$ & $\begin{array}{l}A_{a}(i) \\
\left(\mathrm{s}^{-1}\right)\end{array}$ & $\omega_{K}(i)$ \\
\hline 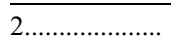 & 1 & 1 & 0 & 0 & $1 s^{2}{ }^{1} S_{0}$ & 0.0000 & & & \\
\hline $2 \ldots \ldots \ldots \ldots \ldots \ldots$ & 2 & 3 & 0 & 2 & $1 s 2 s^{3} S_{1}$ & 7.7331 & & & \\
\hline $2 \ldots \ldots \ldots \ldots \ldots \ldots$ & 3 & 3 & 1 & 0 & $1 s 2 p^{3} P_{0}^{o}$ & 7.7623 & $3.19 \mathrm{E}+08$ & & \\
\hline $2 \ldots \ldots \ldots \ldots$ & 4 & 1 & 0 & 0 & $1 s 2 s{ }^{1} S_{0}$ & 7.7659 & & & \\
\hline $2 \ldots \ldots \ldots \ldots \ldots \ldots$ & 5 & 3 & 1 & 2 & $1 s 2 p^{3} P_{1}^{o}$ & 7.7668 & $6.82 \mathrm{E}+13$ & & \\
\hline $2 \ldots \ldots$ & 6 & 3 & 1 & 4 & $1 s 2 p^{3} P_{2}^{o}$ & 7.7876 & $2.08 \mathrm{E}+09$ & & \\
\hline $2 \ldots \ldots \ldots \ldots \ldots \ldots$ & 7 & 1 & 1 & 2 & $1 s 2 p^{1} P_{1}^{o}$ & 7.8073 & $6.49 \mathrm{E}+14$ & & \\
\hline 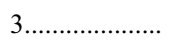 & 1 & 2 & 0 & 1 & $1 s^{2} 2 s^{2} S_{1 / 2}$ & 0.0000 & & & \\
\hline 3..................... & 2 & 2 & 1 & 1 & $1 s^{2} 2 p^{2} P_{1 / 2}^{o}$ & 0.0528 & $2.00 \mathrm{E}+09$ & & \\
\hline 3...................... & 3 & 2 & 1 & 3 & $1 s^{2} 2 p^{2} P_{3 / 2}^{o}$ & 0.0749 & $5.71 \mathrm{E}+09$ & & \\
\hline $3 \ldots \ldots \ldots \ldots \ldots \ldots \ldots$ & 4 & 2 & 0 & 1 & $1 s 2 s^{2}{ }^{2} S_{1 / 2}^{3 / 2}$ & 7.6944 & $2.63 \mathrm{E}+13$ & $1.33 \mathrm{E}+14$ & 0.165 \\
\hline $3 \ldots \ldots \ldots \ldots \ldots \ldots \ldots$ & 5 & 4 & 1 & 1 & $1 s\left({ }^{2} S\right) 2 s 2 p\left({ }^{3} P^{o}\right){ }^{4} P_{1 / 2}^{o}$ & 7.7063 & $7.72 \mathrm{E}+12$ & $2.25 \mathrm{E}+11$ & 0.972 \\
\hline 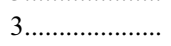 & 6 & 4 & 1 & 3 & $1 s\left({ }^{2} S\right) 2 s 2 p\left({ }^{3} P^{o}\right){ }^{4} P_{3 / 2}^{o}$ & 7.7127 & $2.79 \mathrm{E}+13$ & $1.04 \mathrm{E}+12$ & 0.964 \\
\hline $3 \ldots \ldots \ldots \ldots \ldots \ldots$ & 7 & 4 & 1 & 5 & $1 s\left({ }^{2} S\right) 2 s 2 p\left({ }^{3} P^{o}\right){ }^{4} P_{5 / 2}^{o}$ & 7.7291 & $3.91 \mathrm{E}+05$ & & 1.000 \\
\hline $3 \ldots \ldots \ldots \ldots \ldots \ldots \ldots$ & 8 & 2 & 1 & 1 & $1 s\left({ }^{2} S\right) 2 s 2 p\left({ }^{3} P^{o}\right)^{2} P_{1 / 2}^{o}$ & 7.7508 & $3.73 E+14$ & $3.97 \mathrm{E}+13$ & 0.904 \\
\hline $3 \ldots \ldots \ldots \ldots \ldots \ldots \ldots$ & 9 & 2 & 1 & 3 & $1 s\left({ }^{2} S\right) 2 s 2 p\left({ }^{3} P^{o}\right)^{2} P_{3 / 2}^{o}$ & 7.7657 & $6.61 \mathrm{E}+14$ & $6.00 \mathrm{E}+09$ & 1.000 \\
\hline 3..................... & 10 & 4 & 1 & 1 & $1 s\left({ }^{2} S\right) 2 p^{2}\left({ }^{3} P\right){ }^{4} P_{1 / 2}$ & 7.7711 & $4.06 \mathrm{E}+13$ & $3.56 \mathrm{E}+11$ & 0.991 \\
\hline 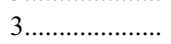 & 11 & 2 & 1 & 1 & $1 s\left({ }^{2} S\right) 2 s 2 p\left({ }^{3} P^{o}\right){ }^{2} P_{1 / 2}^{o}$ & 7.7807 & $3.11 \mathrm{E}+14$ & $6.55 \mathrm{E}+13$ & 0.826 \\
\hline 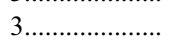 & 12 & 4 & 1 & 3 & $1 s\left({ }^{2} S\right) 2 p^{2}\left({ }^{3} P\right){ }^{4} P_{3 / 2}$ & 7.7830 & $1.47 \mathrm{E}+13$ & $1.88 \mathrm{E}+12$ & 0.887 \\
\hline $3 \ldots \ldots \ldots \ldots$ & 13 & 2 & 1 & 3 & $1 s\left({ }^{2} S\right) 2 s 2 p\left({ }^{3} P^{o}\right){ }^{2} P_{3 / 2}^{o}$ & 7.7841 & $3.16 \mathrm{E}+12$ & $1.04 \mathrm{E}+14$ & 0.030 \\
\hline $3 \ldots \ldots \ldots \ldots \ldots \ldots$ & 14 & 4 & 1 & 5 & $1 s\left({ }^{2} S\right) 2 p^{2}\left({ }^{3} P\right){ }^{4} P_{5 / 2}$ & 7.7923 & $6.26 \mathrm{E}+13$ & $3.05 \mathrm{E}+13$ & 0.672 \\
\hline $3 \ldots \ldots \ldots \ldots \ldots \ldots$ & 15 & 2 & 2 & 3 & $1 s\left({ }^{2} S\right) 2 p^{2}\left({ }^{1} D\right){ }^{2} D_{3 / 2}$ & 7.8108 & $5.05 \mathrm{E}+14$ & $1.25 \mathrm{E}+14$ & 0.802 \\
\hline $3 \ldots \ldots \ldots \ldots \ldots$ & 16 & 2 & 1 & 1 & $1 s\left({ }^{2} S\right) 2 p^{2}\left({ }^{3} P\right){ }^{2} P_{1 / 2}$ & 7.8119 & $9.53 \mathrm{E}+14$ & $1.03 \mathrm{E}+14$ & 0.999 \\
\hline 3....................... & 17 & 2 & 2 & 5 & $1 s\left({ }^{2} S\right) 2 p^{2}\left({ }^{1} D\right){ }^{2} D_{5 / 2}$ & 7.8219 & $2.77 \mathrm{E}+14$ & $1.34 \mathrm{E}+14$ & 0.674 \\
\hline 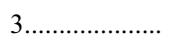 & 18 & 2 & 1 & 3 & $1 s\left({ }^{2} S\right) 2 p^{2}\left({ }^{3} P\right){ }^{2} P_{3 / 2}$ & 7.8370 & $8.60 \mathrm{E}+14$ & $3.72 \mathrm{E}+13$ & 0.959 \\
\hline 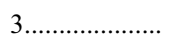 & 19 & 2 & 0 & 1 & $1 s\left({ }^{2} S\right) 2 p^{2}\left({ }^{1} S\right)^{2} S_{1 / 2}$ & 7.8572 & $3.64 \mathrm{E}+14$ & $2.90 \mathrm{E}+13$ & 0.926 \\
\hline
\end{tabular}

Notes.-Table 5 is published in its entirety in the electronic edition of the Astrophysical Journal Supplement. A portion is shown here for guidance regarding its form and content. 
the Supplement. The print versions show data for ions with electron number $N \leq 3$.

It may be seen that in Table 5 levels are identified with the vector $(N, i, 2 S+1, L, 2 J$, and Conf) where $N$ is the electron number, $i$ is the level index, $2 S+1$ is the spin multiplicity, $L$ is the total orbital angular momentum quantum number, $J$ is the total angular momentum quantum number, and "Conf" is the level configuration assignment. For each level, the computed HFR energy and its radiative width $A_{r}(i)$ are listed. For K-vacancy levels, the Auger width $A_{a}(i)$ and the K-shell fluorescence yield $\omega_{K}(i)$ are also given. In Table 6 transitions are identified with the vector $(N, k$, and $i)$ where $k$ and $i$ are the upper and lower level indices, respectively, tabulating its computed wavelength $\lambda$, radiative transition probabilities $A_{r}(k, i)$, weighted oscillator strength $g f(i, k)$, and cancellation factor CF as defined by Cowan (1981).

\section{SUMMARY AND CONCLUSIONS}

Extensive data sets containing energy levels, wavelengths, radiative transition probabilities, absorption oscillator strengths, radiative and Auger widths, and fluorescence yields for K-vacancy levels in the nickel isonuclear sequence have been computed with the atomic structure codes HFR, AUTOSTRUCTURE, and GRASP. For the ionic species $\mathrm{Ni}^{2+}-\mathrm{Ni}^{22+}$, with electron numbers $6 \leq N \leq 26$, this is the first time that such data become available. For ions with $2 \leq N \leq 5$ and $N=27$, detailed comparisons have been carried out with available measurements and theoretical values which bring forth the consistency and accuracy of the present data.

Comparisons of HFR K-level energies with those reported in the NIST database (Ralchenko et al. 2008) for He- and $\mathrm{Li}$-like nickel and the calculated values in $\mathrm{Ni}^{26+}$ by Vainshtein \& Safronova (1985) support an accuracy rating for ions with electron number $N \leq 3$ of better than $3 \mathrm{eV}$. With regards to wavelengths, comparisons between HFR and the tokamak data (Hsuan et al. 1987; Bombarda et al. 1988) for ions with $2 \leq N \leq 5$, between HFR and the solid-state measurements (Hölzer et al. 1997) for $N=27$, and between HFR and other published theoretical values (Vainshtein \& Safronova 1978, 1985; Hsuan et al.
1987; Bombarda et al. 1988) result in a general agreement within $1 \mathrm{~m} \AA$.

Based on the level of agreement of the present HFR $A$-values with those by Vainshtein \& Safronova (1978) and Bombarda et al. (1988) for the highly ionized species $(2 \leq N \leq 5)$, we are confident that they show an accuracy within $10 \%$ for transitions with $A \gtrsim 10^{13} \mathrm{~s}^{-1}$. Regarding radiative widths, similar considerations have led us to the conclusion that our HFR widths are also accurate to $10 \%$. For the $\mathrm{K} \alpha_{2} / \mathrm{K} \alpha_{1}$ and $\mathrm{K} \beta / \mathrm{K} \alpha$ line ratios in the second and third-row ions $(12 \leq N \leq 27)$, the trends along the isonuclear sequence are consistent (within 10\%) for the three independent approaches we have used. For $N=27$, our HFR $\mathrm{K} \alpha_{2} /$ $\mathrm{K} \alpha_{1}$ ratio agrees within 5\% with the solid-state measurements (Salem \& Wimmer 1970; Hölzer et al. 1997), but the HFR K $\beta / \mathrm{K} \alpha$ ratio is $15 \%$ lower than the bulk of the available experimental values located at $0.139 \pm 0.015$.

Comparisons of the HFR Auger widths with those by Bombarda et al. (1988) for species with $3 \leq N \leq 5$ and by Vainshtein \& Safronova (1978) for $N=3$ show a fair consistency for widths greater than $10^{13} \mathrm{~s}^{-1}$, although the latter is higher by $\sim 15 \%$ and the former by $10 \%$ for $N=4$ and $15 \%$ for $N=5$. The systematic deviations with the data by Bombarda et al. (1988) may be due to the orbital optimization procedure they used. Moreover, recent measurements of KLM Auger channel relative intensities (Egri et al. 2008) support our HFR Auger rates. Finally, HFR fluorescence yield and natural K-level width for $N=27$ are consistent within $5 \%$ with the recommended values found in the literature (Hubbell et al. 1994; Campbell \& Papp 2001).

The present radiative and Auger widths will be used in the computations of the K-shell photoionization cross sections of these ions which are required in XSTAR (Kallman \& Bautista 2001) for the modeling of interesting Ni spectral features.

This work was funded in part by the NASA Astronomy and Physics Research and Analysis Program. P. P. and P. Q. are Research Associates of the Belgian FRS-FNRS.
Anders, E., \& Grevesse, N. 1989, Geochim. Cosmochim. Acta, 53, 197

Badnell, N. R. 1986, J. Phys. B, 19, 3827 1997, J. Phys. B, 30, 1

Bautista, M. A., \& Kallman, T. R. 2001, ApJS, 134, 139

Bautista, M. A., Mendoza, C., Kallman, T. R., \& Palmeri, P. 2003, A\&A, 403, 339 2004, A\&A, 418, 1171

Berenyi, D., Hock, G., Ricz, S., Schlenk, B., \& Valek, A. 1978, J. Phys. B, 11, 709

Boirin, L., Parmar, A. N., Barret, D., Paltani, S., \& Grindlay, J. E. 2004, A\&A, 418, 1061

Bombarda, F., Giannella, R., Kallne, E., Tallents, G. J., Bely-Dubau, F. 1988, Phys. Rev. A, 37, 504

Campbell, J. L., \& Papp, T. 2001, At. Data Nucl. Data Tables, 77, 1

Cowan, R. D. 1981, The Theory of Atomic Structure and Spectra (Berkeley: Univ. California Press)

de Plaa, J., Kaastra, J. S., Tamura, T., Pointecouteau, E., Mendez, M., \& Peterson, J. R. 2004, A\&A, 423, 49

Egri, S., Kövér, L., Drube, W., \& Novák, M. 2008, J. Electron Spectrosc. Related Phenom., 162, 115

Eissner, W., Jones, M., \& Nussbaumer, H. 1974, Comput. Phys. Commun., 8, 270

García, J., Mendoza, C., Bautista, M. A., Gorczyca, T. W., Kallman, T. R., \& Palmeri, P. 2005, ApJS, 158, 68

Gastaldello, F., \& Molendi, S. 2004, in The Riddle of Cooling Flows in Galaxies and Clusters of Galaxies, ed. T. Reiprich, J. Kempner, \& N. Soker (Charlottesville: Univ. Virginia), 211, http://www.astro.virginia.edu/coolflow/ Goldstein, G., Huenemoerder, D. P., \& Blank, D. 2004, AJ, 127, 2310

Gorczyca, T. W., Kodituwakku, C. N., Korista, K. T., Zatsarinny, O., Badnell,

N. R., Behar, E., Chen, M. H., \& Savin, D. W. 2003, ApJ, 592, 636

Grant, I. P., \& McKenzie, B. J. 1980, J. Phys. B, 13, 2671

\section{EFERENCES}

Grant, I. P., McKenzie, B. J., Norrington, P. H., Mayers, D. F., \& Pyper, N. C. 1980, Comput. Phys. Commun., 21, 207

Hölzer, G., Fritsch, M., Deutsch, M., Härtwig, J., \& Förster, E. 1997, Phys. Rev. A, 56,4554

House, L. L. 1969, ApJS, 18, 21

Hsuan, H., Bitter, M., Hill, K. W., von Goeler, S., \& Grek, B. 1987, Phys. Rev. A, 35,4280

Hubbell, J. H., et al. 1994, J. Phys. Chem. Ref. Data, 23, 339

Ibarra, A., Matt, G., Guainazzi, M., Kuulkers, E., Jiménez-Bailón, E., Rodriguez, J., Nicastro, F., \& Walter, R. 2007, A\&A, 465, 501

Kaastra, J. S., \& Mewe, R. 1993, A\&AS, 97, 443

Kallman, T., \& Bautista, M. 2001, ApJS, 133, 221

Kallman, T. R., Palmeri, P., Bautista, M. A., Mendoza, C., \& Krolik, J. H. 2004, ApJS, 155, 675

Koyama, K., et al. 2007, PASJ, 59, 245

Markowitz, A., et al. 2007, ApJ, 665, 209

Matt, G., Bianchi, S., Guainazzi, M., \& Molendi, S. 2004, A\&A, 414, 155

Matt, G., \& Guainazzi, M. 2003, MNRAS, 341, L13

McKenzie, B. J., Grant, I. P., \& Norrington, P. H. 1980, Comput. Phys. Commun., 21, 233

Mendoza, C., Kallman, T. R., Bautista, M. A., \& Palmeri, P. 2004, A\&A, 414, 377

Molendi, S., Bianchi, S., \& Matt, G. 2003, MNRAS, 343, L1

Molendi, S., Matt, G., Antonelli, L. A., Fiore, F., Fusco-Femiano, R., Kaastra, J., Maccarone, M. C., \& Perola, C. 1998, ApJ, 499, 608

Palmeri, P., Mendoza, C., Kallman, T. R., \& Bautista, M. A. 2002, ApJ, 577, L119 2003a, A\&A, 403, 1175

Palmeri, P., Mendoza, C., Kallman, T. R., Bautista, M. A., \& Meléndez, M. 2003b, A\&A, 410, 359 
Palmeri, P., Quinet, P., Mendoza, C., Bautista, M. A., García, J., \& Kallman, T. R. 2008, ApJS, 177, 408

Palmeri, P., Quinet, P., Zitane, N., \& Vaeck, N. 2001, J. Phys. B, 34, 4125

Perujo, A., Maxwell, J. A., Teesdale, W. J., \& Campbell, J. L. 1987, J. Phys. B, 20, 4973

Polasik, M. 1998, Phys. Rev. A, 58, 1840

Pounds, K., \& Vaughan, S. 2006, MNRAS, 368, 707

Pounds, K. A., \& Page, K. L. 2005, MNRAS, 360, 1123

Raj, S., Padhi, H. C., Polasik, M., Pawlowski, F., \& Basa, D. K. 2001, Phys. Rev. B, 63, 073109

Ralchenko, Y., Kramida, A. E., Reader, J., \& NIST ASD Team. 2008, NIST Atomic Spectra Database, version 3.1.4 (Gaithersburg: NIST), http://physics .nist.gov/asd3
Rao, N. V., Reddy, S. B., Satyanarayana, G., \& Sastry, D. L. 1986, Physica, $138 \mathrm{C}, 215$

Sako, M., Liedahl, D. A., Kahn, S. M., \& Paerels, F. 1999, ApJ, 525, 921

Salem, S. I., Falconer, T. H., \& Winchell, R. W. 1972, Phys. Rev. A, 6, 2147 Salem, S. I., \& Wimmer, R. J. 1970, Phys. Rev. A, 2, 1121

Scofield, J. H. 1974, Phys. Rev. A, 9, 1041

Sidoli, L., Parmar, A. N., Oosterbroek, T., \& Lumb, D. 2002, A\&A, 385, 940

Slivinsky, V. W., \& Ebert, P. J. 1972, Phys. Rev. A, 5, 1581

Dubau, J., \& Loulergue, M. 1982, J. Phys. B, 15, 1007

Vainshtein, L. A., \& Safronova, U. I. 1978, At. Data Nucl. Data Tables, 21, 49 1985, Phys. Scr., 31, 519

Walter, R., et al. 2003, A\&A, 411, L427 